\title{
Myelinated axons and functional blood vessels populate mechanically compliant rGO foams in chronic cervical hemisected rats
}

\author{
Ana Domínguez-Bajo, ${ }^{1}$ Ankor González-Mayorga, ${ }^{2}$ Carlos R. Guerrero, ${ }^{1}$ F. Javier \\ Palomares, ${ }^{l}$ Ricardo García, ${ }^{1}$ Elisa López-Dolado, ${ }^{2,3}$ and María Concepción Serrano ${ }^{1, *}$ \\ ${ }^{1}$ Materials Science Factory, Instituto de Ciencia de Materiales de Madrid (ICMM), \\ Consejo Superior de Investigaciones Científicas (CSIC), Calle Sor Juana Inés de la Cruz \\ 3, Madrid, 28049, Spain. E-mail: mc.terradas@ csic.es \\ ${ }^{2}$ Laboratory of Interfaces for Neural Repair, Hospital Nacional de Parapléjicos, SESCAM, \\ Finca la Peraleda s/n, Toledo, 45071, Spain \\ ${ }^{3}$ Research Unit of "Design and development of biomaterials for neural regeneration", \\ HNP-SESCAM, Joint Research Unit with CSIC, Spain
}

Keywords: AFM, MRI, nanomechanics, reduced graphene oxide, scaffold, spinal cord injury

Abstract: Neural diseases at the central nervous system including spinal cord injury (SCI) remain therapeutic challenges. Graphene materials are being delineated as alternative tools for neural repair. Herein, the regenerative ability of reduced graphene oxide (rGO) scaffolds to support pivotal features of neural repair at 4 months after SCI is assessed by an interdisciplinary approach. 3D randomly porous foams have been prepared in mechanical compliance with neural cells and tissues (Young's modulus of $1.3 \pm 1.0$ $\mathrm{kPa}$ ) as demonstrated by atomic force microscopy techniques applied ex vivo. After implantation, the significant increase in Young's modulus caused by massive cell/protein infiltration does not alter the mechanical performance of the contralateral spinal cord but provides mechanical stability to the lesion. These aerogels appear fully vascularized and populated with neurites, some of them being myelinated excitatory axons. Clinicallyinspired magnetic resonance imaging studies demonstrate that the scaffolds significantly reduce perilesional damage with respect to rats without implants and cause no compressive damage in the contralateral hemicord and rostral/caudal regions. The rGO 
implants do not either alter the rat spontaneous behavior or induce toxicity in major organs. Finally, preliminary data suggest hints of rGO sheets dissociation and eventual degradation at the injured spinal cord for the first time. In summary, these $3 \mathrm{D}$ porous rGO scaffolds are able to induce, without any further biological functionalization, a compilation of positive effects that have been rarely described before, if ever, for any other material implanted in the injured spinal cord to date.

Since the very first attempts with neurons in $2011,{ }^{[1]}$ it seems more and more reliable that graphene-based materials (GBMs) might provide alternative therapeutic approaches for neural repair. ${ }^{[2]}$ To date, GBMs have been already proved in vitro to stimulate neural differentiation from neural and non-neural progenitor cells, ${ }^{[1,3]}$ to potentiate the sprouting and outgrowth of neurites, ${ }^{[4]}$ to stimulate electrical signalling in neural networks, ${ }^{[5]}$ to reshape neuronal synapses functioning, ${ }^{[6]}$ and to increase neuronal firing by tuning the distribution of extracellular ions, ${ }^{[7]}$ among others. As part of 3D scaffolds, they have also shown positive responses when implanted in the peripheral nervous tissue aiming nerve repair. ${ }^{[8]}$ In the central nervous system (CNS), reduced graphene oxide (rGO) nanosheets behave as permissive materials for neurogenesis when implanted in the olfactory bulb. ${ }^{[9]}$ Interestingly, recent studies have proved the preferential interaction of graphene with cholesterol, potentiating neurotransmission by increasing the number, release probability and recycling rate of synaptic vesicles. ${ }^{[10]}$ Although toxicity concerns are still on stage until the behaviour of GBMs in biological systems becomes completely clear, ${ }^{[11]}$ it seems reasonable the existence of a range of doses, sizes and shapes that could be safely used. ${ }^{[12]}$ Concerns on the biodegradability of these materials also persist, even though evidences of their capacity to undergo biologically-mediated degradative processes in vitro start to 
appear. ${ }^{[13-15]}$ No structural changes or degradation were observed when tested in diverse simulated human gastrointestinal media ex vivo. ${ }^{[16]}$

Scars at the CNS are mainly formed from glial cells (e.g., astrocytes and NG2glia) accompanied by some non-neural cells such as pericytes and meningeal cells, ${ }^{[17]}$ along with collagen and other extracellular matrix components. Importantly, and contrary to scars in other tissues, the CNS tissue significantly softens after injury, both at the neocortex and the spinal cord, as demonstrated by atomic force microscopy (AFM) ${ }^{[18]}$ In this sense, extensive work has already demonstrated that the mechanical properties of the environment play a pivotal role in cell and tissue responses. For instance, neural stem cells fate can be directed by the stiffness of the substrate (preferentially differentiating into neurons in stiffer substrates). ${ }^{[19]}$ Moreover, neurites of dorsal root ganglion cells grow longer on stiffer substrates, ${ }^{[20]}$ while spinal cord astrocytes adapt their morphology to the mechanical properties of the substrate. ${ }^{[21]}$ Thus, it seems reasonable to hypothesize that the reduced ability of neurons to regrow in CNS injuries could be partially associated with changes in the mechanical properties at the lesion site. ${ }^{[18]}$

Spinal cord injury (SCI) continues to be an enormous challenge for scientists and clinicians as there is not an effective therapy to date. Approximately $66 \%$ of the traumatic SCIs in humans occur in the cervical region and $70 \%$ of them are incomplete ${ }^{[22]}$ and lead to chronic sensory-motor upper limb deficits that hinder self-care, independence in the activities of daily living and arm use. Most of these deficits can be attributed to the tracts axotomy, which massively disrupt the descending inputs in all the spinal segments caudal to the lesion epicenter. However, segmental neuronal death also contributes in a great extent to the chronic upper limb deficits in humans, ${ }^{[23]}$ or its homologous forelimb in rats. ${ }^{[24]}$ While the acute SCI phase is characterized by neuronal and glial death, axonal and vascular damage, release of inhibitory neurotransmitters, and ionic disruption, the 
chronic phase is defined by the neurological deficits stabilization. ${ }^{[25]}$ Therefore, it is of great importance to analyze the chronic stage to determine the relative contribution of any proposed therapeutic intervention versus the spontaneous recovery on the functional improvements found. In this context, multidisciplinary efforts are providing encouraging results toward restoring lost functions after SCI, ${ }^{[26,27]}$ including biomaterials. ${ }^{[28-30]}$ In this scenario, recent biomaterials tested range from organic polymers ${ }^{[31,32]}$ to macromolecules including chitosan, ${ }^{[33]}$ and carbon nanotubes, ${ }^{[34,35]}$ to cite a few. Alternatively, and motivated by positive findings regarding the interaction of GBMs with neural cells, in our laboratory we pioneered the exploration of hierarchically channelled 3D rGO scaffolds as advanced platforms to support neural repair after SCI driven by their porosity,

mechanical performance and eventual capacity to undergo electrical stimulation. ${ }^{[36,37]}$ Herein, we demonstrate how randomly porous 3D rGO foams enhance reparative neural responses in long-term chronic hemisected rats (4 months) by means of an interdisciplinary approach including the use of AFM, magnetic resonance imaging (MRI), behavioural tests, standard histological techniques, immunofluorescence studies, confocal laser scanning microscopy, and transmission electron microscopy (TEM). As the CNS is composed of very soft cells with mechanical features similar to elastic solids, ${ }^{[38]}$ these rGO foams are thought to provide a soft scaffolding structure containing mechanical clues in agreement with those of native neural tissues.

\section{Results and discussion}

Fabrication and characterization of mechanically compliant rGO foams

Graphene oxide (GO) slurry used for scaffold fabrication was morphologically characterized by TEM. GO was found to form abundant micron-sized sheets with several 
piled-up layers (Figure 1a). These GO micro-sheets were then dispersed in an aqueous solution and forced to build-up in a 3D structure by simple freeze-casting at $-80^{\circ} \mathrm{C}$. After ice removal by freeze-drying and a subsequent thermal treatment at $200{ }^{\circ} \mathrm{C}$ for $30 \mathrm{~min}$ for GO reduction, rGO micro-sheets assembled into a soft 3D structure with random porosity as characterized by scanning electron microscopy (SEM) (Figure 1b). Interestingly, these foams had partition walls relatively flat and smooth, with transversal sections on the size of hundreds of $\mathrm{nm}$. The high-degree porosity of the resulting structures $(43.87 \pm 3.46 \%$ of porosity and $31.88 \pm 19.43 \mu \mathrm{m}$ of pore size) enable tissue infiltration when implanted. These porous 3D rGO scaffolds, conversely to those previously explored by our group ${ }^{[36,37]}$ avoided the need of hierarchical channels which are scarcely respected by the ingrown tissue when implanted and prevent the necessary transversal regrowth of neurites for propiospinal reconnection.
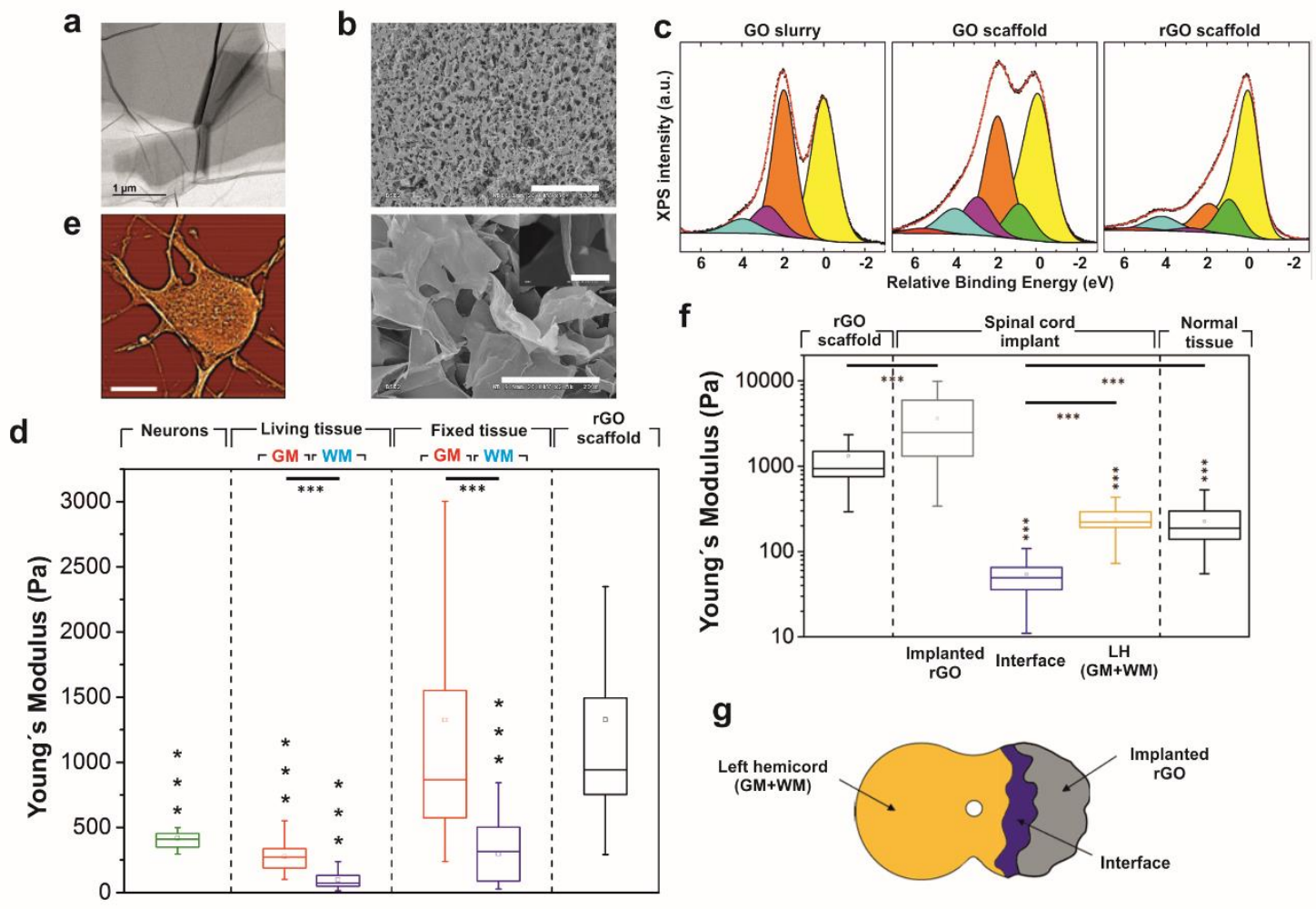

Figure 1. Physic-chemical characterization of rGO foams. (a) Representative TEM image of GO sheets. (b) Morphological characterization of 3D rGO foams by SEM. Scale bars: $500 \mu \mathrm{m}$ (top), $20 \mu \mathrm{m}$ (bottom) and $5 \mu \mathrm{m}$ (inset). (c) C1s XPS spectra corresponding to 
GO slurry, GO scaffold and rGO scaffold together with the fit composed of chemically shifted components associated to different $\mathrm{C}$ chemical bonding, respectively. XPS spectra were normalized to the maximum intensity peak in each case. Data points are represented as black symbols and Shirley background and component peaks using solid lines. The fitting curve (red line) resulted from the addition of contributions belonging to: $\mathrm{C}=\mathrm{C}$ graphitic structures (yellow), $\mathrm{C}-\mathrm{H}$ or $\mathrm{C}-\mathrm{C}$ defective graphitic, $\mathrm{sp} 3$ configurations (green), $\mathrm{C}-\mathrm{OH}$ carbonyl groups (orange), $\mathrm{O}-\mathrm{C}-\mathrm{O}$ carbonyl groups (magenta), $\mathrm{O}-\mathrm{C}=\mathrm{O}$ carboxylic groups (cyan), and $\pi-\pi^{*}$ transitions coming from graphitic structures (red). (d) Nanomechanical characterization of spinal cord tissue (living and fixed), neural cells and rGO scaffolds as obtained from AFM-based measurements (expressed as Young's moduli). (e) Topographical image of the cytoskeleton of a neuron as measured by AFM (scale bar: $10 \mu \mathrm{m}$ ). (f) Nanomechanical characterization of 4-month rGO implants in comparison to rGO scaffold and spinal cord tissue. (g) Scheme of the main regions under study on the rGO-implanted spinal cord tissue. In the plots, statistical significance: $\mathrm{p}<$ $0.001^{* * *}$. Horizontal asterisks refer to comparisons indicated by bars and vertical asterisks refer to comparisons with rGO scaffold. GM: grey matter, LH: left hemicord, WM: white matter.

Comparative X-ray photoelectron spectroscopy (XPS) studies of GO slurry, GO scaffolds and rGO scaffolds confirmed a significant increase in the reduction degree of the samples, accompanied by a decrease of their correspondent $\mathrm{O} / \mathrm{C}$ ratio from 0.84 to $0.28 \%$ (Figure 1c and Table S1). This fact is in line with the signal in the energy region where the photoelectrons from $\mathrm{C}-\mathrm{OH}$ and $\mathrm{O}-\mathrm{C}-\mathrm{O}$ groups are emitted, whose intensity also decreased with the O1s signal. The rGO and GO scaffolds spectra are mainly governed by a high intensity component with asymmetric line shape whose parameter values (peak position and full width half maximum) were obtained by fitting the $\mathrm{C} 1 \mathrm{~s}$ signal from the HOPG reference sample. Therefore, its origin can be certainly ascribed to $\mathrm{sp}^{2}$ bonding. This major asymmetric peak is followed by a symmetric one of much lower intensity $(\sim 15$ and $10 \%$, respectively) shifted $0.85 \mathrm{eV}$ towards higher binding energy. On the one hand, this latter component might be assigned to the presence of a small amount of carbon atoms with $\mathrm{sp}^{3}$ nature due to the observed chemical shift, which is in good agreement to reported values. However, on the other hand, it might also be attributed to disordered regions with $\mathrm{sp}^{2}$ defect-like bonding expected in the outer surface of the sample. Conversely, in the GO slurry, C-C emission is fitted with a single symmetric peak representative of its oxide 
nature. There are also two symmetric contributions related to carbon atoms bound to oxygen at the energy shifted values reported in the literature for hydroxyl $(\mathrm{C}-\mathrm{OH})$, carbonyl $(\mathrm{C}-\mathrm{O})$ and carboxyl $(\mathrm{C}=\mathrm{O})$ groups ${ }^{[39]}$ Finally, the feature at a binding energy of $290.8 \mathrm{eV}$ (broader than any of the previous ones) is clearly associated to the characteristic $\pi-\pi^{*}$ shake-up transition consistent with a majority existence of $\mathrm{sp}^{2}$ configurations in the sample. ${ }^{[39]}$

The mechanical properties of the environment are essential regulators of the biological responses. In this sense, different cells and tissues are characterized by specific ranges of elastic properties, thus making mechanical compliance a pivotal requisite for tissue engineering applications. The elastic response of the rGO scaffolds prepared was quantified by their Young's modulus, $E$, and compared with that from neural cells and spinal cord tissue (Figure 1d). Values were obtained from AFM-based indentation experiments (Figure S1 and S2). ${ }^{[40,41]}$ The macroscopic softness of these rGO scaffolds was confirmed by their Young's moduli (1326 $\pm 1004 \mathrm{~Pa})$. Comparatively, single cortical neurons cultured in vitro were characterized by a ca. 3-fold lower Young's modulus value $(417 \pm 106 \mathrm{~Pa} ; \mathrm{p}<0.001)$, which was itself significantly higher than the values obtained on white and grey matter regions from fresh spinal cord slices (97 \pm 69 and $275 \pm 99 \mathrm{~Pa}$, respectively; $\mathrm{p}<0.001$ ) (Figure $\mathbf{S 3}$ ). These values for spinal cord tissue are in range with those previously reported for the spinal cord (177 and $420 \mathrm{~Pa}$, respectively) and the rat cortical tissue (50 to $500 \mathrm{~Pa}){ }^{[18]}$ When compared to the elastic response of paraformaldehyde-fixed spinal cord tissue (Figure S3), the Young's modulus values of both white and grey matters significantly increased with respect to their fresh counterparts $(296 \pm 211$ and $1324 \pm 1202 \mathrm{~Pa}$, respectively; $\mathrm{p}<0.001)$, as expected from chemically cross-linked tissues, ${ }^{[42]}$ but similar to those obtained for the rGO scaffold in the case of 
grey matter areas $(\mathrm{p}>0.999)$. In any case, the softer nature of the white matter is likely related to its higher content of myelin and other lipid-containing components. ${ }^{[18]}$

Interestingly, somata of pyramidal neurons display elastic modulus between 480 $\mathrm{Pa}$ at $30 \mathrm{~Hz}$ and $970 \mathrm{~Pa}$ at $200 \mathrm{~Hz}$, while values in astrocytes, even though being softer, reach $300 \mathrm{~Pa}$ at $30 \mathrm{~Hz}$ and $520 \mathrm{~Pa}$ at $200 \mathrm{~Hz} \cdot{ }^{[38]}$ Somata of retinal neurons have values as high as $1590 \mathrm{~Pa}$ at $200 \mathrm{~Hz}$. In other tissues, for instance, fibroblasts display an elastic storage value as high as $\sim 5 \mathrm{kPa}$ at $0.1-4.0 \mathrm{~Hz},{ }^{[41]}$ dramatically higher than the elastic modulus of our rGO scaffolds. Moreover, these rGO foams are significantly softer than most of the biomaterials implanted at the injured spinal cord to date, even hydrogels, which account for an additional major drawback that is their fast in situ degradation. Some examples include porous methacrylate hydrogels $(2.9 \pm 0.4 \mathrm{kPa}$ perpendicular to pores and $6.7 \pm 1.8 \mathrm{kPa}$ along pores), ${ }^{[43]}$ cholesterol-modified superporous methacrylate hydrogels $(10.1 \pm 1.8 \mathrm{kPa}),{ }^{[44]}$ protein-functionalized methacrylate scaffolds $(263 \pm 13$ $\mathrm{kPa}),{ }^{[45]}$ ordered porous polycaprolactone scaffolds $(2.1 \mathrm{MPa}),{ }^{[46]}$ and polycaprolactone/gellan gum blends $(79.7 \pm 7.6 \mathrm{kPa}),{ }^{[47]}$ to cite a few. Even though all these biomaterials have reported favourable regenerative responses such as axon growth at the injured spinal cord, no data exist on the potential mechanical distress caused by their mechanical mismatch with the neural tissues.

\section{Preservation of spontaneous behaviour features in chronic rGO-implanted rats}

We then proceeded to implant these 3D rGO scaffolds in acute C6 hemisected rats, immediately after removal of the right C6 hemicord. A consistent anatomo-functional spinal cord correlation has been extensively reported to correlate the specific control of every muscle with each particular spinal segment. This fine topographical organization can predict the deficits according to the injury level. ${ }^{[48]}$ As expected, this SCI model 
caused a significant initial impairment of the ipsilateral forelimb and both hindlimbs. Later on, while both hindlimbs (but mainly the left one) progressively recovered to functional levels, the ipsilateral impaired forelimb remained damaged for the 4-month period analysed. ${ }^{[49]}$ In order to evaluate the degree of focal functional affectation and its progression over time, two tests involving the specific use of forelimbs and characteristic of the spontaneous rat behaviour were selected: grooming and forelimb asymmetry tests. These tests are quite comparable to the clinical tools used to evaluate functional independence for daily living activities in SCI patients. ${ }^{[50]}$ Interestingly, despite the impairments caused by the lesion, animals showed no significant differences in the time spent at either grooming $(p=0.768)$ or naturally exploring the environment $(p=0.903)$ once the injury-induced spinal cord shock was resolved (Figure S4). In all groups, the left forepaw (LFP) retrieved the maximum score (i.e., 5), while injured animals displayed a marked but not statistically significant impartment in the use of the right forepaw (RFP) at 120 days post-injury (DPI) $(3.83 \pm 0.98$ for injured rats without scaffolds - I rats, 3.20 \pm 1.30 for $r G O$ and $5.00 \pm 0.00$ for control rats; $\mathrm{p}=0.432$ and $\mathrm{p}=113$, respectively to control) (Figure 2a). The functional RFP impairment was only statistically significant at 10 DPI $(\mathrm{p}=0.024)$. We next quantified the rate of the different grooming positions reached by both forelimbs, with special attention to positions 3 to 5 and back side (Figure 2b). All injured animals groomed significantly less intensively with the RFP at the most challenging positions ( 5 and back side) than control rats $(p=0.002$ and $p=0.024$, respectively to control). Importantly, rats carrying rGO scaffolds were unable to reach positions further than the eyes area (i.e., position 3) at earlier time points (10 and $30 \mathrm{DPI}$ ). In any case, animals retained the ability to throw the RFP to reach positions 3 and 4 , but typically failed to lift it high enough to reach position 5 or further and make contact with the body surface (Figure $2 \mathrm{~d}$ and 2e). Position 5 and the right back side were scarcely 
explored during the grooming time (Figure $2 \mathrm{f}$ and $2 \mathrm{~g}$ ). To compensate for RFP deficits, all injured animals overused the LFP to reach position 3, although this difference was not statistically significant $(\mathrm{p}=0.146)$.

a

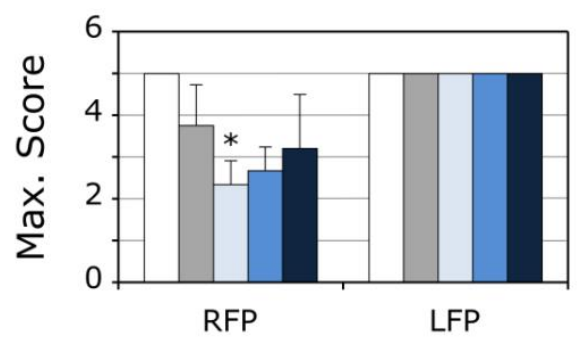

b

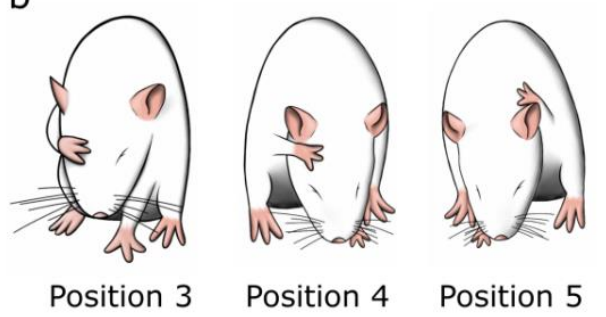

$\mathrm{C}$

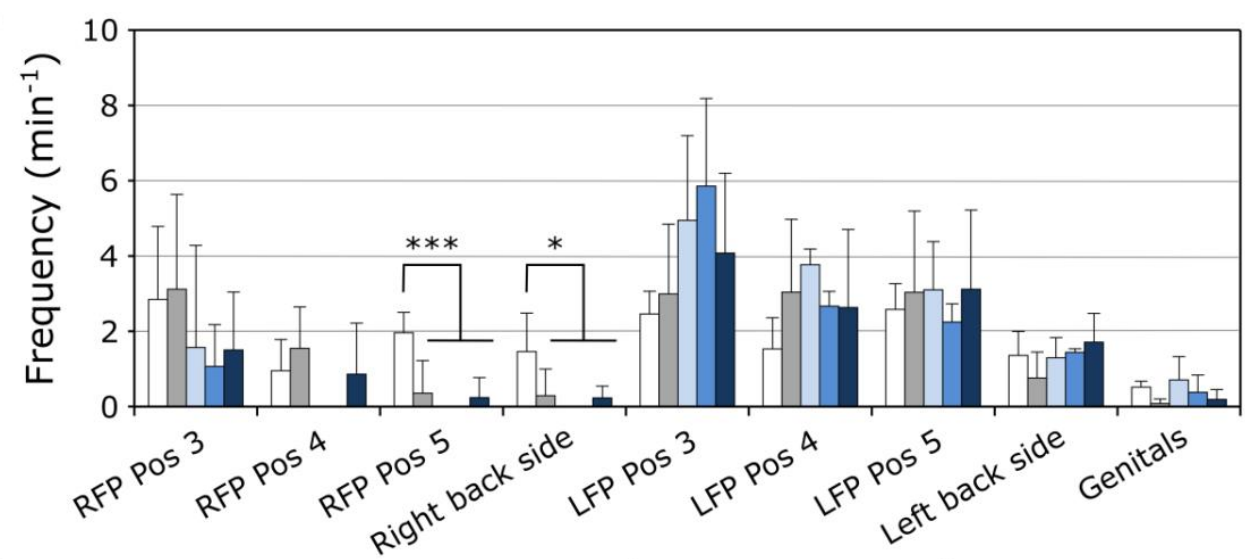

d

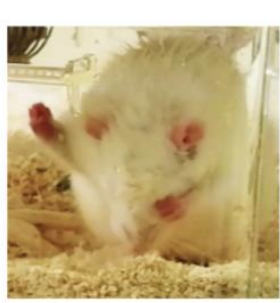

e

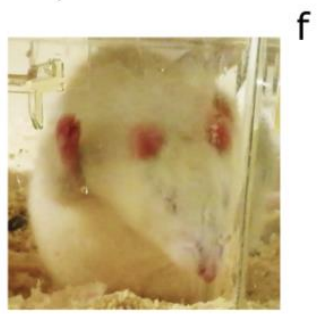

$f$ max

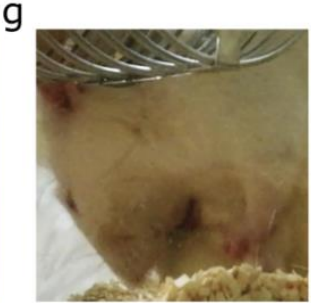

$\mathrm{h}$
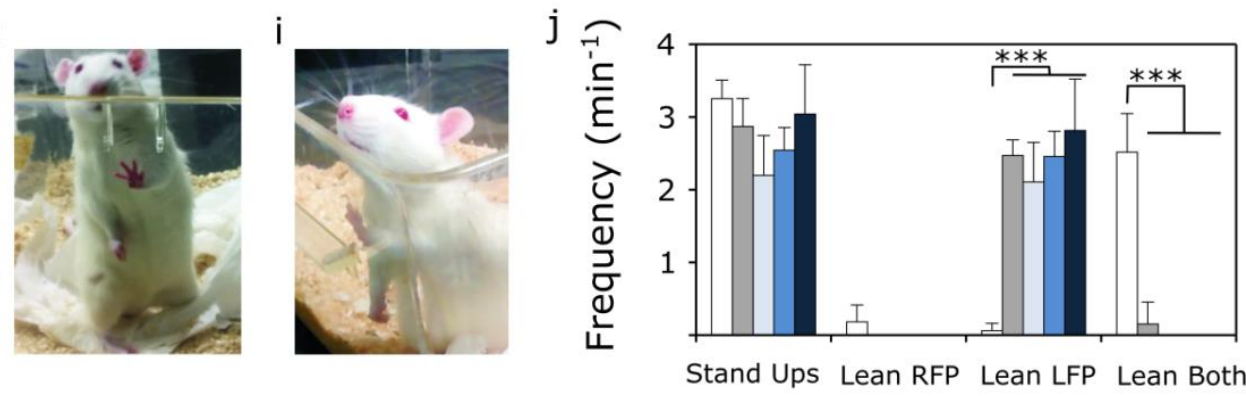

Control $\square$ Injury 120 dpi $\square$ RGO-SC 10 dpi $\square$ RGO-SC 30 dpi

RGO-SC 120 dpi

Figure 2. Behavioral tests on $r G O$ rats as measured by grooming (a-g) and asymmetry (h-j) tests. (a) Maximum score numbers achieved for the RFP and LFP during grooming. (b) Scheme of the relevant grooming positions. (c) Frequency of the different positions reached for the RFP and LFP during grooming. (d,e) Representative photographs of $r G O$ rats failing positions 4 and 5 with the RFP, respectively. (f,g) Representative photographs of $r G O$ rats attaining position 5 with the RFP and grooming on the right back side, respectively. (h,i) Frontal and lateral views of a representative $r G O$ rat leaning on the 
cage wall, respectively. (j) Frequency of stand-ups and supports on RFP, LFP and both forepaws during asymmetry tests. Statistics: $p<0.05^{*}$ and $\mathrm{p}<0.005^{* * *}$. DPI: days postinjury; LFP: left forepaw; Pos: position; RFP: right forepaw.

Asymmetry tests revealed that both groups of injured animals avoided leaning on their RFP, solving the necessity of body support by almost exclusively resting on the open LFP palm (Figure $2 \mathrm{~h}$ and 2i). In these rats, the LFP use was therefore significantly prioritized over the use of both paws typical of control animals $(p<0.001$; Figure $2 j)$. No statistically significant differences were found on the frequency of stand-ups among groups $(p=0.272)$, neither on the use of the RFP $(p=0.164)$. All together, the acute and chronic functional impairments caused by the unilateral hemisection practiced in this study were in agreement with previous findings in cervical SCI. ${ }^{[49,51]}$ Despite these findings, we do not discard minor motor behavioural improvements to have also occurred as studies regarding upper limbs in humans evidence that clinical tests may fail discriminating subtle functional changes. ${ }^{[52]}$

\section{Outstanding MRI findings of 4-month $r G O$ implants}

A clinically inspired MRI protocol was used to analyse the whole cervical spinal cord enlargement (i.e., metameres between the caudal part of $\mathrm{C} 4$ and the caudal part of $\mathrm{T} 1$ ) in an attempt to: (1) have a "contextualized" morphological vision of the lesion epicenter with and without implants and (2) search for radiological signs of damage not directly caused by the hemisection along all the metameres responsible for the rat forelimb functions. In all animals, the lesion epicenter was located in the right C6 hemicord and affected it almost entirely (Figure 3a). Its dimensions were significantly larger in rats receiving $\mathrm{rGO}$ scaffolds, both as the mean maximum transverse diameter $(2.40 \pm 0.06$ $\mathrm{mm}$ in $r G O$ and $1.06 \pm 0.14 \mathrm{~mm}$ in $I$ rats; $\mathrm{p}=0.008)$ and the mean lesion volume $(26.41$ $\pm 3.24 \mathrm{~mm}^{3}$ in $r G O$ and $6.29 \pm 1.28 \mathrm{~mm}^{3}$ in $I$ rats; $\mathrm{p}=0.008$ ) (Figure $3 \mathrm{~b}$ ). No radiological 
signs of either syringomyelia or hematomyelia were found in any injured animals. Slight left displacements relative to the midline were found in the coronal sections of $r G O$ rats $(0.26 \pm 0.11 \mathrm{~mm})$, suggesting a possible "mass effect" of the scaffold (Figure 3c). However, no signs of compressive damage were detected in either the contralateral hemicord or the rostral/caudal regions. Differential features in the MRI signal intensity at the interface zone, eventually related to foreign body reactions, could not be identified. The interface volume was indirectly quantified in the $r G O$ group by subtracting the volume of the scaffold itself from the total volume of the whole lesion area. Interestingly, the mean volume of the interface was not higher than the volume of the lesion itself in the $I$ group $\left(5.30 \pm 3.25 \mathrm{~mm}^{3}\right.$ versus $6.29 \pm 1.28 \mathrm{~mm}^{3}$, respectively; $\left.\mathrm{p}=0.784\right)$. Therefore, most of the lesion volume increment in $r G O$ rats was primarily due to the scaffold itself but not to an increment of the surrounding interface.

Areas of perilesional damage were always identified in the ipsilateral side of the spinal cord in both groups (Figure 3c), both above or below the lesion, at a similar distance from the lesion epicenter $(2.64 \pm 0.85 \mathrm{~mm}$ in $I$ rats and $1.92 \pm 0.53 \mathrm{~mm}$ in $r G O$ rats; $\mathrm{p}=0.566)$ and with a similar extension along the coronal sections $(2.76 \pm 0.38 \mathrm{~mm}$ in $I$ rats and $1.89 \pm 0.72 \mathrm{~mm}$ in $r G O ; \mathrm{p}=0.286)$. Nevertheless, the mean damage surface was significantly larger in rats without $\mathrm{rGO}$ scaffolds $\left(0.95 \pm 0.29 \mathrm{~mm}^{2}\right.$ in $I$ and $0.18 \pm$ $0.03 \mathrm{~mm}^{2}$ in $\left.r G O ; \mathrm{p}=0.005\right)$, corresponding to hyper-intense areas suggestive of gliosis. ${ }^{[53,54]}$ Finally, a similar mean MRI signal intensity was measured in both groups $(1.70 \pm 0.26$ in $I$ and $1.48 \pm 0.49$ in $r G O ; \mathrm{p}=0.102)$, thus suggesting the same type of phenomenon taking place in both groups of injured rats but being more dramatic in those animals without rGO implants. These MRI findings permit to accurately interpret the behavioural tests results, supporting the circumscribed affectation of the metameres involved in the right forelimb movements observed at 4 months. 

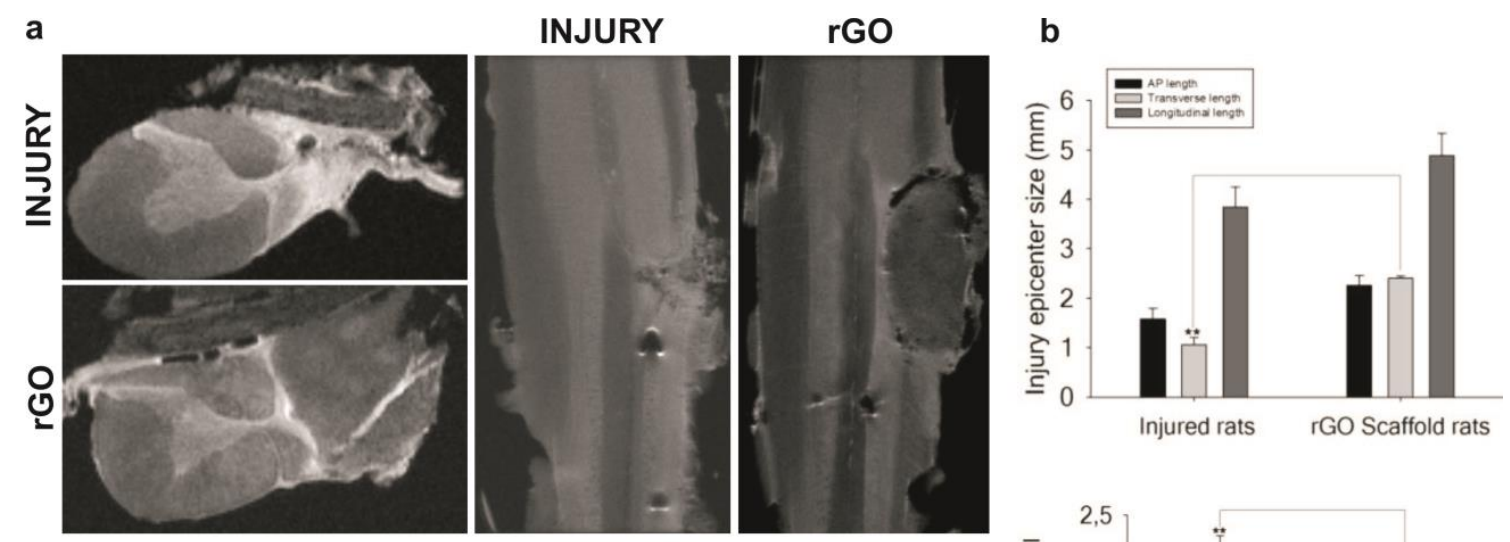

C

INJURY

rGO
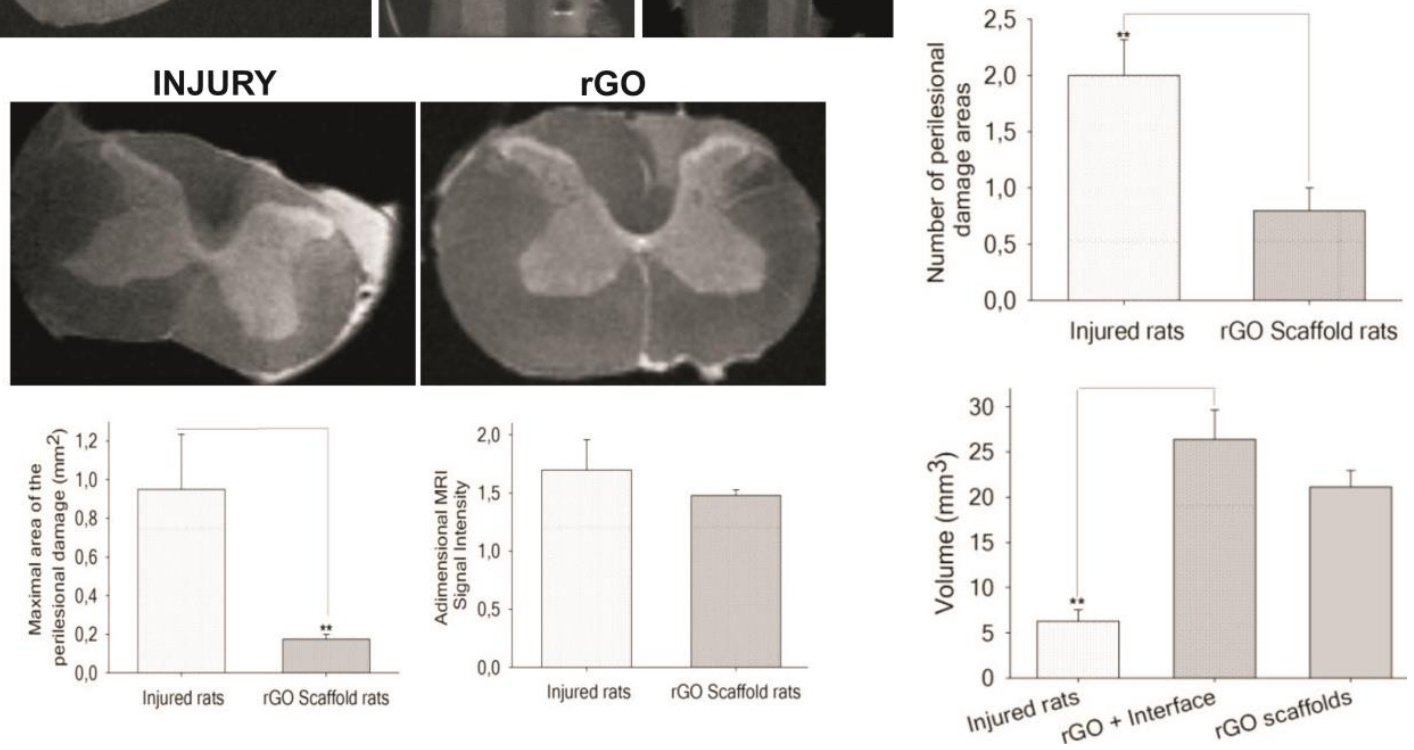

Figure 3. MRI features of the rGO implants. (a) Representative spinal cord MRI images corresponding to: axial (left column) and coronal (right column) scans of $I$ and $r G O$ rats. (b) Quantifications of de lesion epicenter size and volume and the number of perilesional damage areas. (c) Axial scans of representative perilesional damaged areas in both $I$ and $r G O$ rats and respective quantifications.

\section{rGO foams promote collagen deposition and provide structural support to the lesion}

Contrary to lesions at the brain and likely related to its softer nature, injuries at the spinal cord typically result in irregular and diffuse boundaries with a varying degree of tissue damage among animals. ${ }^{[18]}$ This softening is also significant outside of the lesion site. These features add both uncertainty in defining the size/location of the lesion and an extensive variability to the data. In our case, this effect was especially evident in the case of injured rats without rGO scaffolds. Conversely, the presence of the rGO scaffold 
clearly assisted the structural stabilization of the lesion site and diminished these phenomena.

By using a conventional Masson's trichrome staining, we identified the infiltration of both cells and collagen fibers in both groups (Figure S5a), although largely more abundant within the rGO foams implanted. More importantly, the chronic evolution of the injury revealed significant differences in the area occupied by cavities, with a $29.4 \pm$ $10.3 \%$ of empty areas in $I$ rats and as low as $5.6 \pm 2.8 \%$ in $r G O$ animals as quantified from histological sections $(\mathrm{p}=0.023)$. When visualized by TEM (Figure S5b), lesion areas holding rGO foams were found highly compact, with rare free space but abundant cells and deposited collagen fibers. rGO sheets forming the foam walls were clearly identifiable. On the contrary, the injury site in $I$ rats was characterized by profuse empty space and disorganized tissue elements. When estimated from TEM images at low magnification $(2,000 \mathrm{X})$, a significantly different distribution of grey-scale pixels was detected between groups, with a predominant peak in the whitish region corresponding to pure resin without biological content in rats without implants (Figure S5c; maximum peaks location: pixels 78 and 163 for $r G O$ and 158 and 224 for $I$ rats; statistically significant differences between groups at pixels 3-34, 90-195, and 205-254, at least $\mathrm{p}<$ $0.05)$.

\section{rGO foams transmit mechanically compliant signals to the injured spinal cord tissue}

AFM-indentation measurements were then carried out on the spinal cord tissue containing the rGO scaffolds implanted for 4 months (Figure 1e). Young's modulus values are provided for the main regions of the sample as depicted in the scheme (Figure 1f). As expected from damage in the $\mathrm{CNS}^{[18]}$ the interface tissue showed a significantly lower Young's modulus $(54 \pm 29 \mathrm{~Pa} ; \mathrm{p}<0.001)$. However, the lesion site carrying the rGO 
scaffolds displayed the significantly highest Young's modulus $(3624 \pm 2664 \mathrm{~Pa}$; p < 0.001), even when compared to the rGO scaffolds before implantation (1326 $\pm 1004 \mathrm{~Pa})$, likely due to collagen deposition and massive cell infiltration of the foam. Nonetheless, and importantly, this decrease in the elasticity of the right hemicord holding the scaffold was not transmitted to the left counterpart spinal tissue, as its elastic response was found in range with the one found in the fresh control spinal cord tissue $(237 \pm 66$ and $225 \pm$ $109 \mathrm{~Pa}$, respectively; $\mathrm{p}=0.366$; control spinal cord tissue calculated as the average of the values for grey and white matters). These mechanical findings are in agreement with the absence of both perilesional/compressive damage in surrounding spinal cord regions observed by MRI and alterations in the rat spontaneous behaviour.

\section{Reduction in macrophages and vimentin ${ }^{+}$cells infiltrating rGO foams}

The first observable consequence at the cellular level of the lesion practised was a significant increase in cell density both at the lesion epicentre and, to a lower extent, in its caudal and rostral interfaces in all injured animals as demonstrated by Hoechst staining (Figure S6; $\mathrm{p}<0.001$ ). Most of these lesion-infiltrating cells might have been recruited by the release of chemotactic messengers from blood vessels in the surroundings but also resulted from the proliferation of local cells at the injured spinal cord at early times of the lesion. Indeed, we already observed significant cell colonization of comparable rGO scaffolds as early as $10 \mathrm{DPI},{ }^{[36]}$ which were maintained at $30 \mathrm{DPI} .{ }^{[37]}$ When compared with injured rats without scaffolds, two major significant features were identified. First, the presence of the rGO scaffold significantly decreased cell density at the lesion site (p $<0.001$ ). Second, the absence of the scaffold ( $I$ rats) slightly increased cell density in perilesional areas at 1-2 $\mathrm{mm}$ from the lesion border (PL12) with respect to their correspondent left hemicord ( $\mathrm{LH} ; \mathrm{p}=0.023)$. 
We next investigated the presence of specific markers for major features of native and injured neural tissue by immunostaining and confocal laser scanning microscopy (CLSM, Figure 4). Control and $I$ rats were processed and analysed in parallel with $r G O$ rats for comparisons (Figure S7). First, neural components were visualized by labelling for map-2 and tau, two major cytoskeleton proteins expressed by neurons. No statistically significant differences were found in either LH or PL12 $(p=0.589$ for map-2 and $p=$ 0.051 for tau at LH; $p=0.347$ for map- 2 and $p=0.741$ for tau at PL12). However, the interface tissue, both rostral and caudally (RIF and CIF, respectively), experienced a significant decrease in the presence of map- 2 and tau in $r G O$ rats $(\mathrm{p}<0.02$ for CIF and RIF with respect to map-2 LH; $p<0.009$ for CIF and RIF with respect to tau LH). These differences were also evidenced in $I$ rats, except for the presence of tau at RIF which remained similar to the $\mathrm{LH}$ ( $\mathrm{p}<0.003$ for CIF and RIF with respect to map-2 LH; $p=$ 0.004 for CIF and $p=0.069$ for RIF with respect to tau LH). In any case, the presence of both proteins was comparable in both regions for both treatment groups (map-2: $p=0.613$ for CIF and $p=0.512$ for RIF; tau: $p=0.124$ for CIF and $p=0.510$ for RIF). However, at the lesion site, all injured animals experienced a dramatic reduction of both neural markers when compared to LH ( $\mathrm{p}<0.001)$. Nonetheless, the presence of limited but detectable positive labelling at the lesion site for these two markers deserved further investigation as will be explained later. 
a

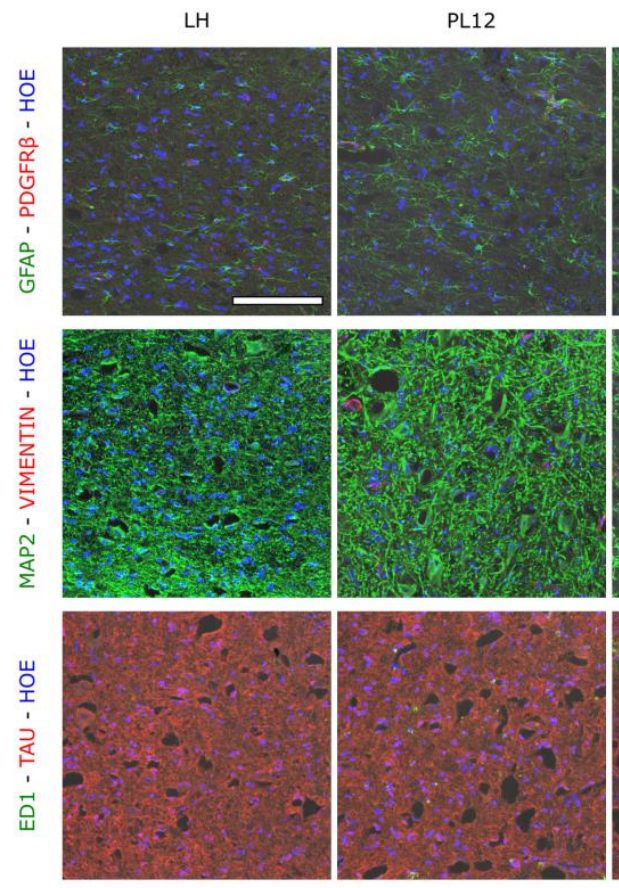

CIF
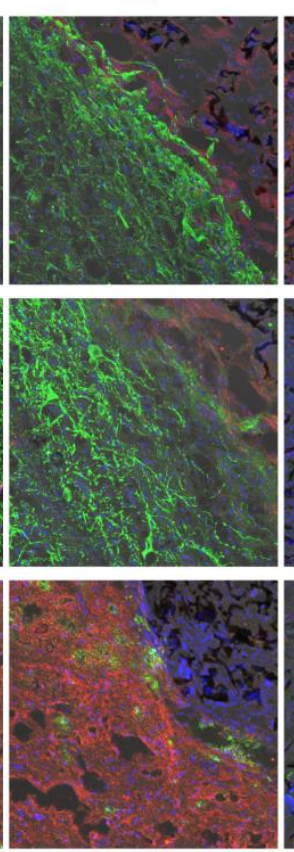

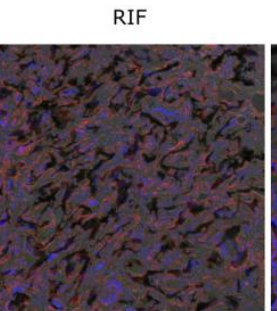

Lesion
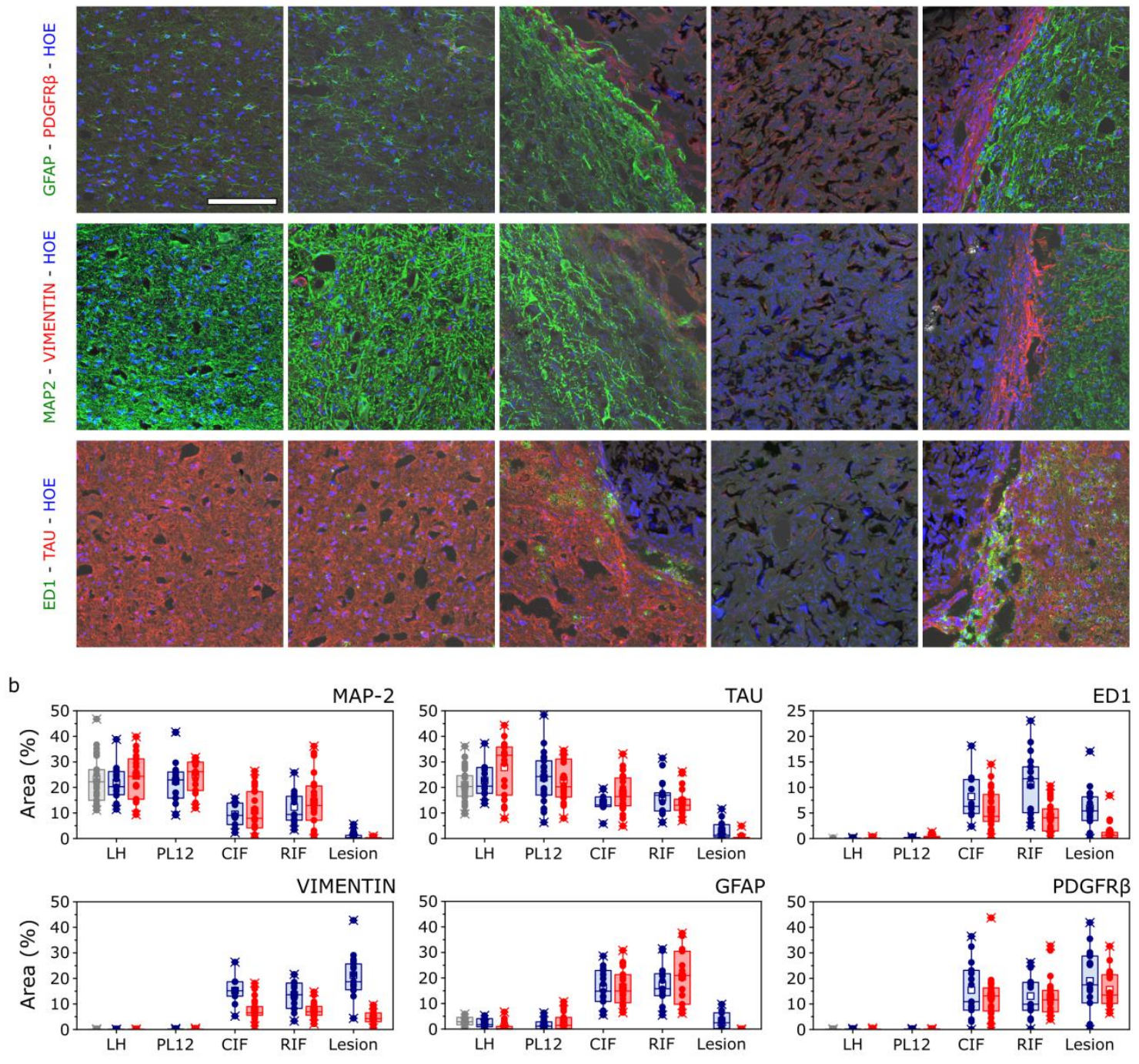

- Control

- Injury

- rGO

Figure 4. Immunofluorescence characterization of the lesion site in $r G O$ rats. (a) Representative CLSM images for the different markers and areas under study. Scale bar: $150 \mu \mathrm{m}$. (b) Quantitative data from immunofluorescence images. CIF: caudal interface; LH: left hemicord; PL12: perilesional areas at 1-2 mm from the lesion border; RIF: rostral interface.

The presence of activated astrocytes labelled as $\mathrm{GFAP}^{+}$cells was significantly restricted to the interface area $(\mathrm{p}<0.001$ with respect to LH, PL12 and lesion areas for both CIF and RIF), without evidences of significant penetration into the rGO foam ( $\mathrm{p}=$ 0.081 with respect to $\mathrm{LH}$ ), as previously reported for biocompatible block copolymers of poly-lactic-co-glycolic acid and poly-l-lysine. ${ }^{[31]}$ Conversely, a significant amount of $\mathrm{GFAP}^{+}$cells were found at the lesion site in $I$ rats $(\mathrm{p}=0.002)$. $\mathrm{ED}^{+}$cells accounting for 
macrophages were also significantly abundant at both CIF and RIF in both $I$ and $r G O$ rats ( $p<0.003$ with respect to LH and PL12). The amount of macrophages did not differ at CIF between $I$ and $r G O$ rats $(\mathrm{p}=0.095)$, but did at PL12 $(\mathrm{p}=0.003)$ and RIF $(\mathrm{p}=0.001)$. More importantly, the presence of the rGO implants dramatically reduced the amount of macrophages invading the lesion site with respect to $I$ rats $(\mathrm{p}<0.001)$, being just slightly above values found at the $\mathrm{LH}(\mathrm{p}=0.042)$. In agreement with these results, conducting polymer hydrogels based on tannic acid and polypyrrole also lowered the amount of ED1 ${ }^{+}$ cells within the lesion site after six weeks of implantation at the hemisected thoracic spinal cord. ${ }^{[55]}$

The interface tissue in both $I$ and $r G O$ rats was also characterized by the significant presence of cells positive for vimentin and PDGFR $\beta$ ( $p \leq 0.001$ for both CIF and RIF with respect to LH and PL12). Vimentin, but not PDGFR $\beta$, was significantly less abundant at the lesion site than at the interface (vimentin: $\mathrm{p}=0.027$ with respect to $\mathrm{CIF}$ and $p=0.010$ with respect to RIF; PDGFR $\beta: p=0.764$ with respect to CIF and $p=0.585$ with respect to RIF). There were no significant differences attributed to the presence of the rGO foam for PDGFR $\beta$ in any of the areas under study $(p>0.05)$, but there were for vimentin at both interfaces and the lesion site $(\mathrm{p}<0.002)$. In injured animals without rGO scaffolds, the abundance of these markers was only significantly reduced with respect to the lesion site in the case of RIF for vimentin (vimentin: $p=0.266$ with respect to CIF and $p=0.049$ with respect to RIF; PDGFR $\beta$ : $p=0.906$ with respect to CIF and $p=0.502$ with respect to RIF). Remarkably, at the lesion site, vimentin but not PDGFR $\beta$ was significantly reduced by the presence of rGO (vimentin: $\mathrm{p}<0.001$; PDGFR $\beta$ : $\mathrm{p}=0.309$ ). We hypothesize that cells positive for these two markers might be having a positive role at the lesion site, as vimentin is considered a marker of immature glial cells and new-born neural stem cells ${ }^{[56]}$ and PDGFR $\beta$ is expressed by highly proliferative type A pericytes, 
essential to regain tissue integrity after SCI. ${ }^{[17]}$ In any of the cases, LH in animals carrying rGO foams showed no statistically significant differences when compared with undamaged grey matter areas in control rats except for a slight decrease in GFAP ( $p=$ $0.001)$.

\section{Ingrowth of myelinated vGlut $2^{+}$axons within rGO scaffolds}

To further elucidate the presence of map2 ${ }^{+}$and tau ${ }^{+}$elements within the rGO foams, two additional markers were investigated: $\beta$-III tubulin and SMI311, specifically binding to proteins contained in cytoskeleton microtubules and neurofilaments, respectively. Interestingly, positive staining for both markers was found in all five animals carrying rGO scaffolds dedicated to these studies (Figure 5a). 3D-plots more clearly illustrate the significant colonization of the scaffold by $\beta$-III tubulin ${ }^{+}$and $\mathrm{SMI} 311^{+}$neurites (Figure 5b). A representative PL12 region was plotted for comparison. Curiously, when images for both markers were merged, elements uniquely stained for either $\beta$-III tubulin or SMI311were identified (Figure 5c), as well as double-stained neurites. When quantified, neurites re-growth within the $\mathrm{rGO}$ foams accounted for $5.42 \pm 1.75 \mathrm{~mm}$ per $\mu^{2}$ of scaffold for $\beta$-III tubulin and $3.71 \pm 2.57 \mathrm{~mm}$ per $\mu \mathrm{m}^{2}$ for SMI311. Previous work with other materials such as chitosan hydrogels have also reported axonal growth through the lesion site when functionalized with neurotrophin-3, accompanied by functional MRI and magnetic resonance diffusion tensor imaging studies. ${ }^{[33]}$ The predominant expression of $\beta$-III tubulin against other neurite markers is not surprising as, during mammalian and avian development, this protein is considered the earliest neuron-associated cytoskeletal marker. ${ }^{[57,58]}$ Importantly, its expression is maintained in mature neurons and confers microtubules a more dynamic nature, likely related to neurites in the process of development and growth. ${ }^{[59]}$ The presence of both markers at the LH and PL12 was not 
significantly affected by the rGO implants (Figure S8; $p>0.05$ ). However, the lesion reduced, although not significantly, the presence of both proteins at the interface ( $p$ > 0.05), both rostral and caudally, in a similar magnitude to that found in injured animals not carrying rGO foams $(\mathrm{p}>0.05)$.
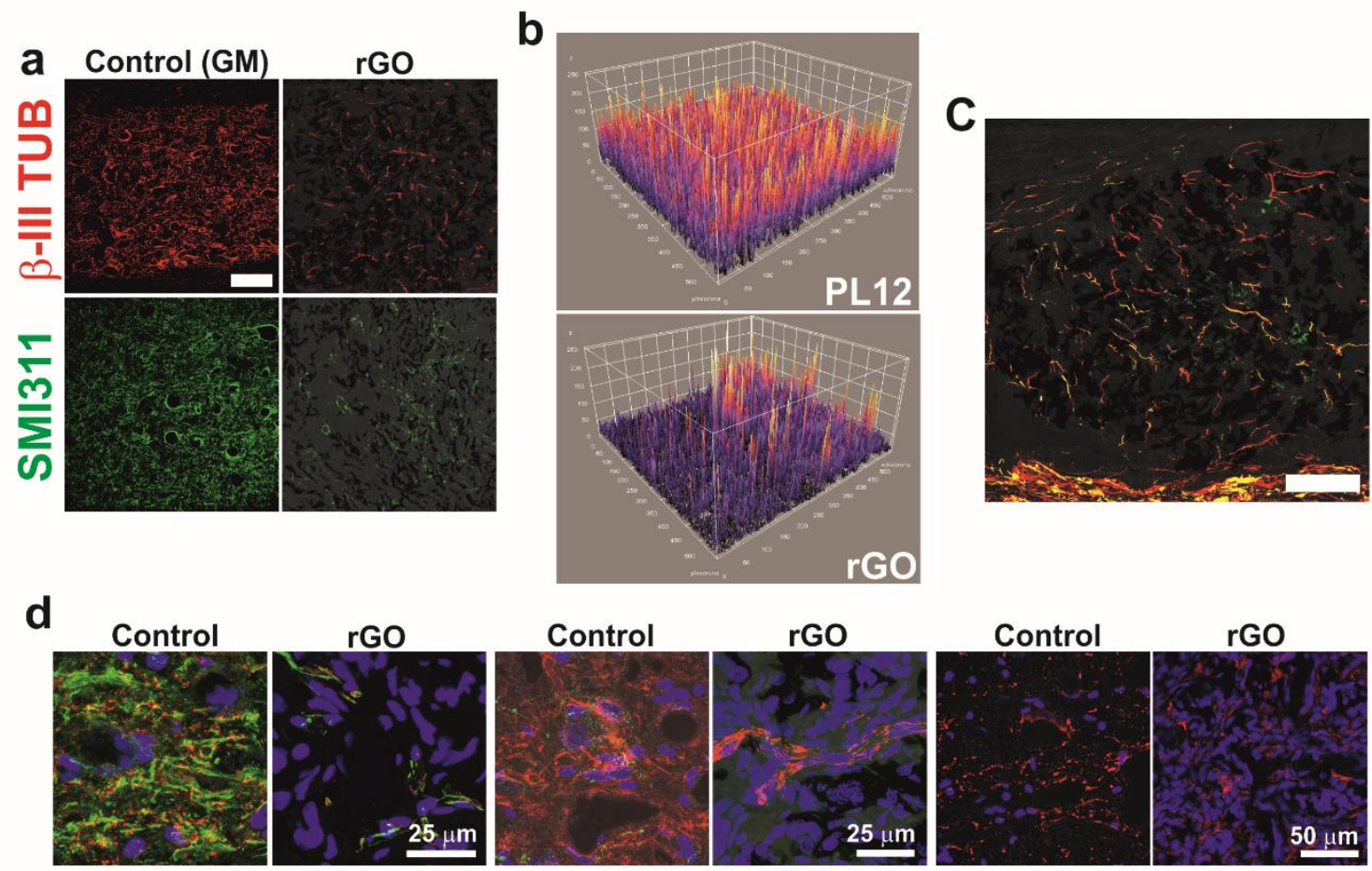

rGO

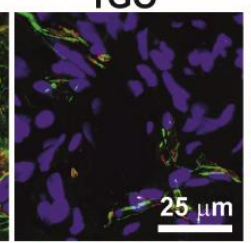

Control

rGO

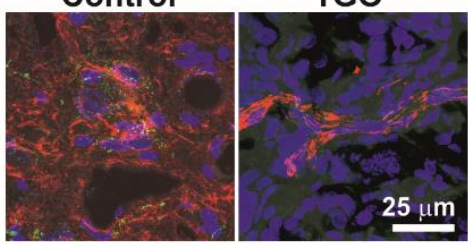

$\beta$-III TUB - TH

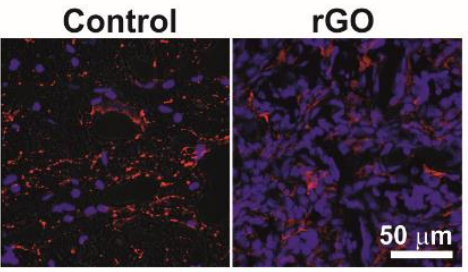

5-HT
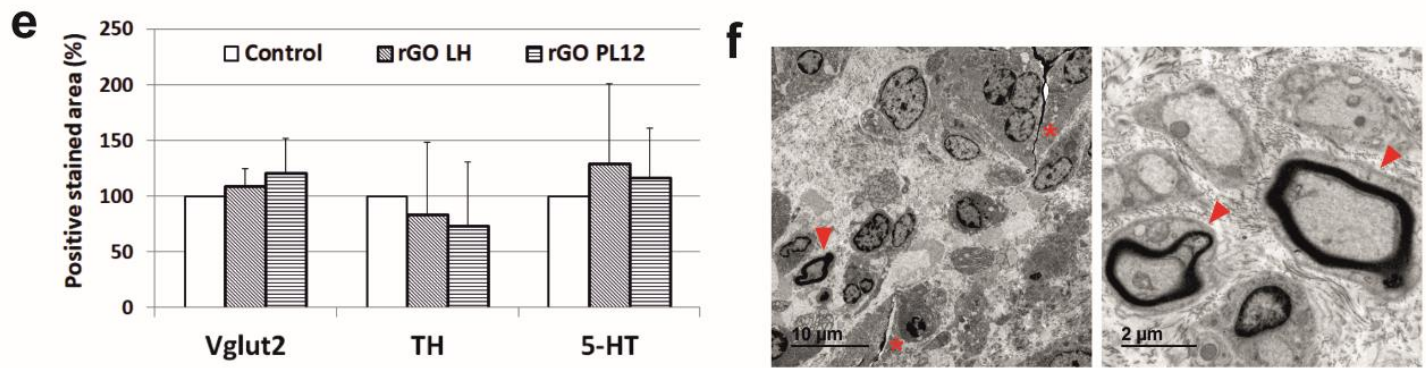

Figure 5. Axon growth at the lesion site in $r G O$ rats. (a) Representative CLSM images for $\beta$-III tubulin and SMI311. GM in control rats is shown for comparison. Scale bars: $150 \mu \mathrm{m}$. (b) 3D reconstruction plots for $\beta$-III tubulin in PL12 and $r G O$ (image area 555.61 $\mu \mathrm{m} \times 555.61 \mu \mathrm{m}$ ). (c) Illustrative CLSM image merging $\beta$-III tubulin and SMI311 labeling. Scale bar: $150 \mu \mathrm{m}$. (d) Representative CLSM images for Vglut2, TH and 5-HT in control (healthy grey matter) and $r G O$ (lesion site containing rGO scaffolds) rats. Counterstaining with SMI311 and $\beta$-III tubulin for neurites location. Cell nuclei stained with Hoechst. (e) Quantitative data for Vglut2, TH and 5-HT at LH and PL12 in comparison to GM in control rats (healthy spinal cord tissue). (f) Representative TEM images evidencing the presence of myelinated axons (red arrow heads) within rGO scaffolds (red asterisks). GM: grey matter; LH: left hemicord; PL12: perilesional areas at 
1-2 $\mathrm{mm}$ from the lesion border; TH: tyrosine hydroxylase; Vglut2: vesicular glutamate transporter 2; 5-HT: serotonin.

We next investigated the chemical nature of these new neurites by looking at specific neurotransmitters. The presence of vesicular glutamate transporter 2 (Vglut2) was used for identifying excitatory glutamatergic fibers, tyrosine hydroxylase for excitatory dopaminergic fibers and serotonin for inhibitory serotoninergic ones (Figure 5d). Grey matter of control rats (uninjured) were used for comparison. Neurites growing within the rGO foams were found predominantly positive for Vglut2. Tyrosine hydroxylase, if present, was negligible. Regarding serotonin, although a positive labelling was observed within the scaffold, neither the morphology of the staining nor the association with $\beta$-III tubulin ${ }^{+}$neurites reflected mature serotonin fibers, so we attributed this finding to either non-specific binding sites for the antibody or early immature serotoninergic fibers. Importantly, when the presence of all three neurotransmitters systems was studied in PL12 and LH in $r G O$ rats, no statistically significant changes were detected in comparison to control rats in any case (Figure 5e; Vglut2: $107.8 \pm 17.0 \%$ and $120.1 \pm 31.9 \%, \mathrm{p}=0.467 ; \mathrm{TH}: 82.5 \pm 65.9 \%$ and $72.6 \pm 57.5 \%, \mathrm{p}=0.746 ; 5-\mathrm{HT}: 128.8$ $\pm 72.0 \%$ and $115.4 \pm 45.6 \%, \mathrm{p}=0.713$, respectively for PL12 and LH). Finally, detailed morphological TEM analysis of the ingrown neurites within the rGO foams revealed the existence of organized multi-layer myelin coatings in some of these neural elements (Figure 5f).

Functional recovery in incomplete SCI is associated to neuroanatomical plasticity processes, which comprise sprouting of spared fibers (compensatory plasticity), sprouting and regrowth of severed fibers (regenerative plasticity) and plastic adaptations in both supraspinal and intraspinal neural circuits. ${ }^{[60]}$ Specifically, spared reticulospinal fibers are able to initiate compensatory adaptations by spontaneous sprouting below the lesion at 
the lower thoracic cord. ${ }^{[61]}$ Besides, propiospinal neurons also display high intrinsic potential for neuroanatomical adaptations, ${ }^{[62]}$ participating in the formation of propiospinal detour pathways. ${ }^{[63]}$ They are thought to participate in the compensatory mechanisms for motor function restoration in forelimbs, especially in the case of finger function. ${ }^{[64]}$ The newly-grown axons found within these rGO scaffolds might have origin in brainstem nuclei, more largely devoted to forelimb rather than hindlimb motor neurons. ${ }^{[65]}$ The abundance of Vglut $2^{+}$labelling could be in agreement with this brainstem nuclei origin for those, as glutamate (in particular the Vglut2 system) is the predominant transmitter pathway in the brainstem neurons responsible for excitatory motor commands. ${ }^{[66]}$ These results support the hypothesis of rGO foams reinforcing intrinsic plastic adaptations in preexisting reticulo-propiospinal connections after cervical injuries.

\section{Formation of new functional microvessels within rGO implants}

As blood supply is essential for tissue repair and maintenance and the absence of blood supply is known as one of the major limitations after $\mathrm{SCI},{ }^{[30]}$ we then specifically investigated the abundance of blood vessels within and around the implanted rGO foams by analysing two specific proteins: (1) Reca-1, as a marker of mature vascular endothelial cells, and (2) laminin, as a major component of the basal lamina in vasculature (Figure 6). Importantly, a significant amount of Reca-1 was detected within the rGO foams, thus demonstrating the existence of new small-sized blood vessels (i.e., microvessels) likely formed from pre-existing ones at the interface (angiogenesis). Specifically, an average of $109.47 \pm 19.43$ sectioned vascular structures per $\mathrm{mm}^{2}$ of scaffold, with an area of $1.02 \pm$ $0.55 \cdot 10^{4} \mu \mathrm{m}^{2}$ per $\mathrm{mm}^{2}$ and a diameter of $10.16 \pm 6.21 \mu \mathrm{m}$ were colonizing the interior areas of the scaffold. Similarly, Reca- $1^{+}$blood vessels grew and branched within methacrylate-based hydrogels, with and without incorporation of basic fibroblast growth 
factor, at 8 weeks after implantation at the injured thoracic spinal cord. ${ }^{[67]}$ Further studies with hyaluronic acid hydrogels modified with an anti-Nogo receptor antibody and loaded with poly(lactic-co-glycolic acid) microspheres containing brain-derived neurotrophic factor and vascular endothelial growth factor also reported a large number of new blood vessels and regenerated nerve fibers within and around the implants. ${ }^{[68]}$ Although the number of vascular structures within our rGO scaffolds was significantly inferior to that found in control grey matter $(284.20 \pm 41.21 ; \mathrm{p}<0.001)$, their diameter was similar $(9.07$ $\pm 6.34 \mu \mathrm{m} ; \mathrm{p}=0.202$ ). Interestingly, the area positively stained for Reca-1 did not significantly vary with respect to the control grey matter at any other location including LH, PL12, CIF, and RIF ( $\mathrm{p}>0.05)$.
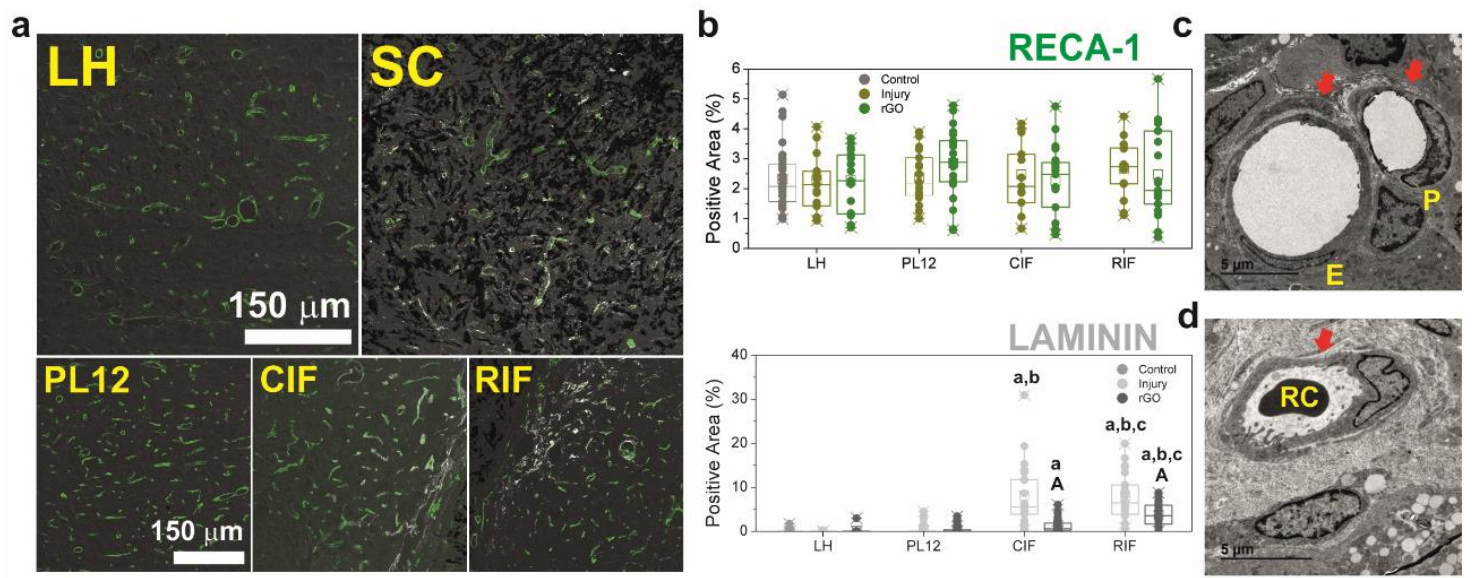

Figure 6. Analysis of microvessels formation in $r G O$ rats. (a) Representative immunofluorescence images for Reca-1 and laminin at the different areas under study. (b) Quantitative data from immunofluorescence images for both markers. Values for control and injured rats without scaffolds are shown for comparison. CIF: caudal interface; LH: left hemicord; PL12: perilesional areas at 1-2 mm from the lesion border; RIF: rostral interface. Statistics: significant differences with respect to (a) LH, (b) PL12, (c) CIF, and (A) I group (p < 0.05). Representative TEM images illustrating (c) the wall structure of the microvessels formed (red arrows) containing mature endothelial cells (E) and pericytes $(\mathrm{P})$ and $(\mathrm{d})$ their functional nature demonstrated by the presence of red blood cells in their lumen (RC).

When focused on laminin, a slight but not significant increase of this protein was found within the scaffold and PL12 in comparison to LH, with an area of positive staining 
of $0.63 \pm 1.14$ and $0.58 \pm 1.06 \%$, respectively $(p>0.05)$. However, laminin at the interface tissue around the scaffold dramatically augmented ( $\mathrm{p} \leq 0.002$ for both CIF and RIF with respect to $r G O \mathrm{LH})$, likely serving to guide the new blood vessels to the foam. In this sense, the augmentation in extracellular matrix components including laminin, mainly associated to basal laminae, are known to upregulate after injury, ${ }^{[69]}$ probably related to the destruction of blood vessels caused by the lesion. ${ }^{[18]}$ As RIF experienced a more remarkable increase in laminin than CIF $(\mathrm{p}=0.001)$, we hypothesize a preferential origin of blood vessels from pre-existing ones at a rostral location. Laminin expression in $I$ rats was markedly higher than on $r G O$ rats, although heterogeneous, both around and inside the lesion site $(\mathrm{p} \leq 0.001)$. Conversely to rGO-implanted rats, the augmentation of laminin inside the lesion in $I$ animals was similar to that found at the interfaces $(p>0.05)$ and significantly higher than at the correspondent LH $(\mathrm{p}<0.001)$. Both collagen and laminin, identified in the lesion site in $r G O$ rats, are likely driving new microvessels from pre-existing ones to grow throughout the lesion site. This angiogenic phenomenon and subsequent vascular remodelling is of essential relevance for neural repair at the damaged CNS, as recently demonstrated in the brain for hyaluronic acid hydrogels implanted after stroke. ${ }^{[70]}$

Detailed analysis by TEM demonstrated small-sized vascular elements (mainly capillary but also some terminal arterioles and venules based on size and wall structure), adequately structured within the rGO foams and containing easily identifiable endothelial cells and supportive pericytes around them (Figure 6c). Moreover, it revealed the presence of blood components inside these vascular elements invading the 3D rGO foam (Figure 6d), thus confirming their functioning as blood suppliers to the interior areas of the scaffold. This significant angiogenesis is hypothesized to play an essential role in the neurite regrowth found, in agreement with recent findings after stroke. ${ }^{[70]}$ 


\section{Preliminary insights of rGO foam degradation after 4 months in the injured spinal cord}

Biodegradation of implantable devices is an attractive feature frequently pursued. ${ }^{[71]}$ Regarding GBMs, recent work with human myeloperoxidase and degranulating human neutrophils point out towards graphene not to be biopersistent. ${ }^{[14]}$ Despite this progress, GBMs degradation in in vivo scenarios continues to be poorly understood, with no reports to date demonstrating any insights of graphene degradation when implanted at the CNS. In this study, TEM was used to identify both dissociation and biodegradation features in the rGO foams implanted. High-magnification TEM images first evidenced qualitative changes in the degree of compaction of rGO sheets on the foam walls (Figure 7a; 300,000X). Comparisons among rGO foams before and after progressive times of implantation (i.e., 10, 30 and 120 days) revealed a dramatic and significant increase of the thickness of the foam walls $(r G O: 84.1 \pm 35.6 \mathrm{~nm}$; $r G O-10$ DPI: $90.2 \pm 46.3 \mathrm{~nm}, \mathrm{p}=$ 0.789 with respect to $\mathrm{rGO} ; r G O-30 \mathrm{DPI}: 108.4 \pm 51.5 \mathrm{~nm}, \mathrm{p}=0.005$ with respect to $\mathrm{rGO}$ and $\mathrm{p}=0.097$ with respect to $r G O-10 \mathrm{DPI} ; r G O-120 \mathrm{DPI}: 138.0 \pm 54.6 \mathrm{~nm}, \mathrm{p}<0.001$ with respect to $\mathrm{rGO}, \mathrm{p}<0.001$ with respect to $r G O-10 \mathrm{DPI}$ and $\mathrm{p}=0.004$ with respect to rGO-30 DPI; Figure 7b), accompanied by a clear fading of their colour intensity. Individualized small fragments of rGO sheets were even visible in the surroundings of the walls (red asterisks). When estimated as the variation in colour (Figure 7c), significant changes in the distribution of grey-scale pixels were observed with the time of implantation (maximum peaks location: 229 for rGO, 221 for $r G O-10 \mathrm{DPI}, 206$ for $r G O$ 30DPI, and 202 for $r G O-120 \mathrm{DPI}$; statistically significant differences among groups at pixels $85-200$ and 215-250, at least $\mathrm{p}<0.05)$. The observed shift of black pixels to lighter values is attributed to the progressive disassembly of rGO sheets in the foams, while the 
shift of white ones to darker pixels correlates with an increase in the degree of tissue ingrowth in the foam. Both shifts were accompanied by an expected increase of intermediate grey values. Besides dissociation of the scaffold structure, rGO fragments were frequently found intracellularly. Hints of attempts of internalization by phagocytosis-like mechanisms were noticed (Figure 7d). Internalized rGO was sometimes included in intracellular compartments (Figure 7e), or directly in contact with cytoplasmic components (Figure $7 \mathrm{f}$ and $7 \mathrm{~g}$ ). Disassembly of rGO sheets was also noticed within cells (Figure 7h). Further quantitative studies by techniques such as Nuclear Magnetic Resonance and Raman spectroscopy might be helpful to identify chemical changes in rGO compatible with biodegradation processes. Preliminary studies in our laboratory by XPS and IR spectroscopy and microscopy failed to detect any chemical changes undoubtedly attributed to biodegradation at the injured spinal cord (data not shown). In any case, degradative action by enzymes and phagocytic immune cells seems pivotal for rGO sheets biodegradation. ${ }^{[14]}$ Specifically, neutrophils have the capacity to degrade GO generating degradation products that seem non-genotoxic. ${ }^{[72]}$ Importantly, mechanisms of cellular uptake for graphene nanomaterials remain unclear. Previous in vitro findings have evidenced the entrance of $350 \mathrm{~nm}$ graphene flakes by active filopodia of macrophages while larger ones $(2 \mu \mathrm{m})$ are able to directly trespass cell membranes perpendicularly. ${ }^{[73]}$ In a different work, $500 \mathrm{~nm}$-sized GO flakes entered by clathrinmediated endocytosis and larger ones $(1 \mu \mathrm{m})$ underwent phagocytosis in $\mathrm{C} 2 \mathrm{C} 12$ mouse mesenchymal stem cells in vitro. ${ }^{[74]}$ 

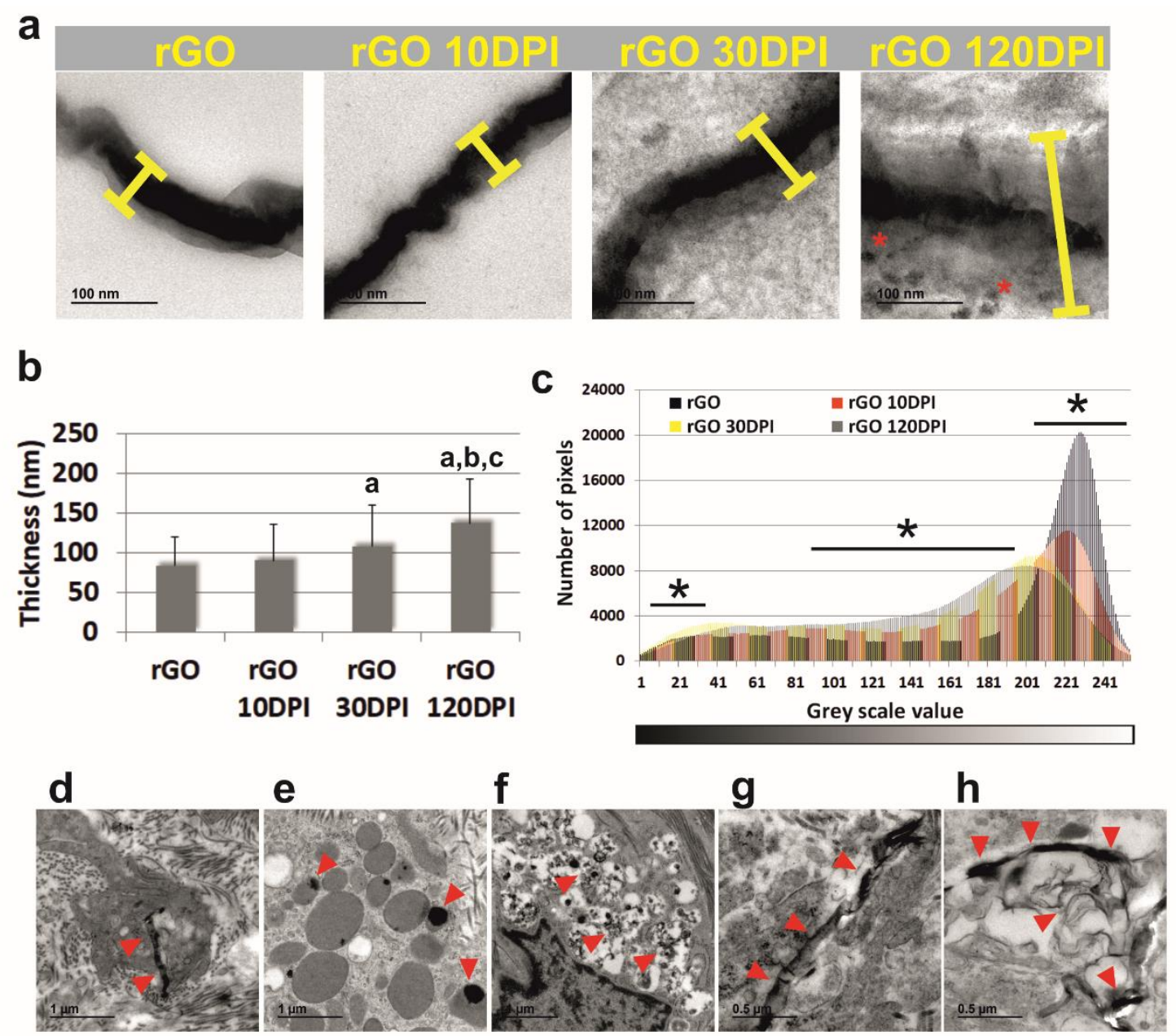

Figure 7. Preliminary scaffold disassembly and biodegradation features in $r G O$ rats by TEM. (a) Representative high-magnification images illustrating details of rGO foams over implantation time. Yellow lines indicate rGO scaffold wall thickness and red asterisks point out dissociation clues in their immediate periphery. (b) Wall thickness variation over time. Statistics: significant differences with respect to (a) $\mathrm{rGO}$, (b) $r G O$ 10DPI, and (c) $r G O$ 30DPI (p < 0.05). (c) Pseudo-quantitative estimations of rGO scaffold disassembly by analyses of the variations in grey-scale pixels of TEM images. Statistics: $\mathrm{p}<0.05^{*}$. (d-h) Specific morphological features of internalized rGO fragments indicative of eventual biodegradation processes ongoing (red arrow heads).

\section{Absence of toxicity insights in major organs}

As previous work with GBMs has demonstrated accumulation and eventual granuloma formation in liver, spleen and lungs, ${ }^{[75-77]}$ we then inspected major organs for toxic effects

(Figure 8). Although scaffold implantation takes place at the injured spinal cord, identified disassembly processes could eventually drive fragments of our rGO scaffolds to the blood stream. Anatomophatological studies revealed no signs of either macroscopic or microscopic organ damage in any of the tissues analysed (i.e., kidney, liver, lung, 
spleen) at this time point. Respective organ-specific cells in all cases were found morphologically identical to those of control rats by careful hematoxylin/eosin staining and TEM inspection. This absence of organ toxicity is not surprising as the dose used in our case $\left(250 \mu \mathrm{g}\right.$ per rat, equivalent to $\left.\sim 700 \mu \mathrm{g} \mathrm{Kg}^{-1}\right)$ was largely below that found toxic by others $\left(400 \mu \mathrm{g}\right.$ per animal in mice, ${ }^{[75]} 10 \mathrm{mg} \mathrm{kg}^{-1}$ in mice/rats, ${ }^{[76]}$ and $1-10 \mathrm{mg} \mathrm{Kg}^{-1}$ in mice $^{[77]}$ ) thus supporting the hypothesis of a dose-dependence toxicity already defined for GBMs. ${ }^{[12]}$ Nonetheless, further studies at longer times of implantation are required to discard systemic toxicity of these materials and their degradation products.
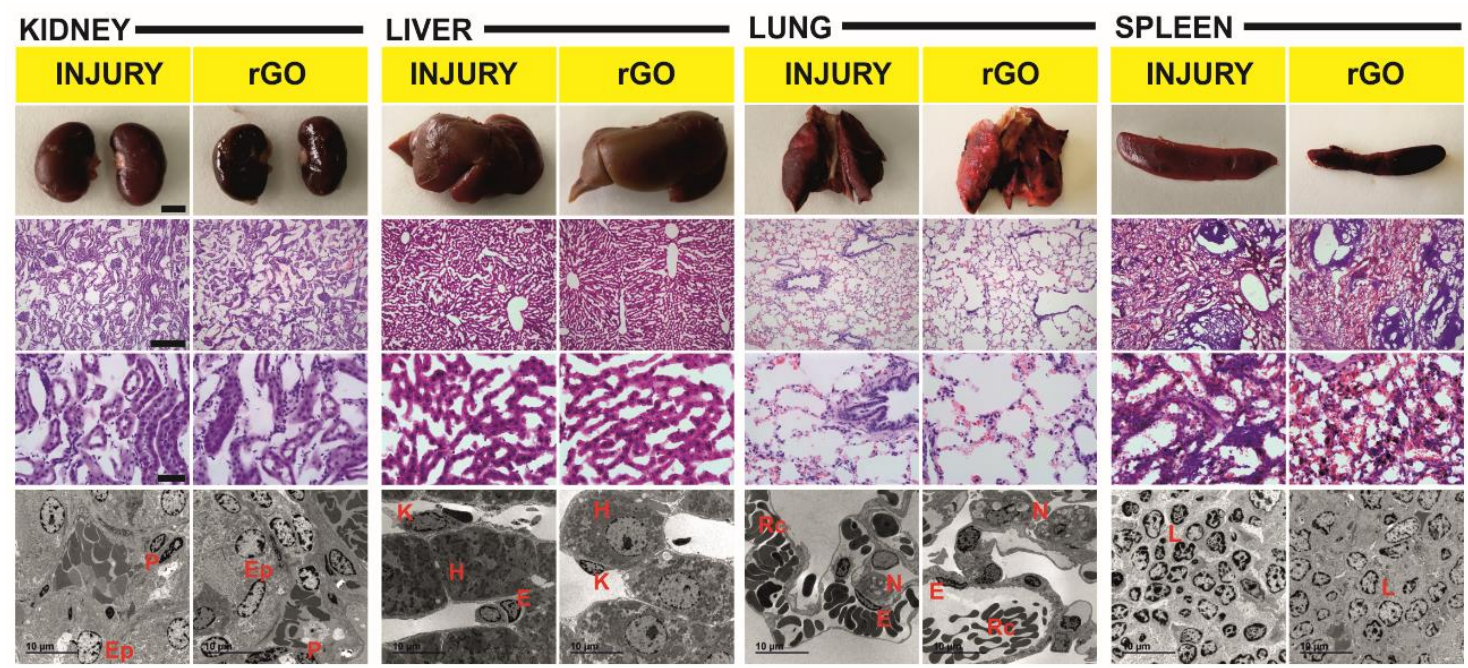

Figure 8. Histopathological and tissue structural effects in key organs (kidneys, liver, lungs and spleen) after 4-month rGO scaffold implantation at the injured spinal cord. Representative images from macroscopic views (top), H\&E staining (middle) and TEM analyses (bottom). Scale bars: $2 \mathrm{~mm}, 250 \mu \mathrm{m}, 50 \mu \mathrm{m}$, and $10 \mu \mathrm{m}$, respectively. Images from organs in hemisected rats without rGO scaffolds ( $I$ rats) are shown for comparison. E: endothelial cell; Ep: epithelial cell; H: hepatocyte; K: Kuffer cell; L: lymphocytes; N: neumocyte; P: podocyte; Rc: red blood cell.

\section{Conclusion}

Based on the relevance of mechanical compliance and the softness of the spinal cord tissue, we herein focused on 3D soft rGO aerogels for neural repair after SCI motivated by the previous encouraging responses of GBMs with neural cells and tissues. 
In the absence of any additional biological functionalization or drug-loading, these foams mediated neural repair at the injured spinal cord by permitting excitatory myelinated axons to grow through the lesion site assisted by functional blood microvessels indicative of microvascular remodeling. These rGO foams clearly benefited the stabilization of the damage by avoiding scar retraction within the lesion site and decreasing perilesional areas of gliosis both above and below the SCI epicenter. Preliminary results by TEM evidenced, for the first time, ultrastructural changes compatible with ongoing disassembly and eventual degradative processes of the rGO sheets at the injured spinal cord. Finally, neither toxic responses in major organs nor changes in the spontaneous forelimb motor behaviour were induced by the chronic implantation of these rGO foams at 4 months. Future studies at longer time points will deepen on the in vivo degradation of rGO at the neural tissue, eventual systemic responses of toxicity derived and neuronal tracing for identifying the origin and destination of neurites populating the rGO foams.

\section{Methods}

Material: GO slurry was purchased from Graphenea, S.A. (Batch \#C1250/GOB125/D; 4,6 wt $\%$ concentration, $>95 \%$ monolayer content). Neural cell culture media and supplements were acquired from Fisher Scientific. Chemical reagents were purchased from Sigma-Aldrich. Specific commercial details of antibodies are provided in Table S2. All reagents were used as received unless otherwise indicated.

Animals for scaffold implantation: Adult male Wistar rats were provided by the animal facilities of the Hospital Nacional de Parapléjicos. All the experimental protocols for surgical scaffold implantation at the spinal cord adhered to the regulations of the European Commission (directives 2010/63/EU and 86/609/EEC) and the Spanish 
government (RD53/2013 and ECC/566/2015) for the protection of animals used for scientific purposes.

Preparation of implantable rGO scaffolds: 3D rGO scaffolds were fabricated by a freezecasting methodology. Briefly, GO slurry was gently dispersed in distilled water (5 wt $\%$ ) and pipetted into tap covers of $0.5 \mathrm{ml}$ Eppendorf tubes $(50 \mu \mathrm{l})$. Samples were then frozen at $-80^{\circ} \mathrm{C}$ overnight and subsequently freeze-dried for $24 \mathrm{~h}$. The resulting cylindrical and porous monoliths were thermally treated at $200{ }^{\circ} \mathrm{C}$ for $30 \mathrm{~min}$. Prior to implantation, scaffolds were sterilized under UV radiation and spontaneously infiltrated with sterile tissue culture grade water at $4{ }^{\circ} \mathrm{C}$ for $48 \mathrm{~h}$.

Physic-chemical characterization of $G O$ slurry and $r G O$ scaffolds: TEM studies were performed by using a Jeol JEM 1010 microscope (Tokyo, Japan) at 80-100 kV with a coupled digital camera (Gatan SC200, Pleasanton, CA, USA) for image acquisition. For SEM studies, a Hitachi S-3000N microscope working at $15 \mathrm{kV}$ with secondary electrons was used. XPS spectra were acquired at normal emission in an UHV chamber with a base pressure of $10^{-10}$ mbar equipped with a hemispherical electron energy analyzer (SPECS Phoibos 150 spectrometer) and a 2D delay-line detector, using a Mg Al-Ka (1253.6 eV) X-ray source. For experimental details on XPS studies, please refer to the Supplementary Material.

rGO scaffold implantation in hemisected rats: Adult male rats were used for scaffold implantation at the age of $20.0 \pm 2.9$ weeks $(360.0 \pm 35.8 \mathrm{~g}$ in weight $)$. Rats were housed at a $12 \mathrm{~h}$ light/dark cycle with food and water ad libitum. The amount of rGO implanted was $250 \mu \mathrm{g}$ per animal $\left(\sim 700 \mu \mathrm{g} \mathrm{kg}^{-1}\right)$. The lesion model of choice was a cervical unilateral hemisection at the right $\mathrm{C} 6$, rostral to the bulk of triceps brachii motoneurons. The rationale behind the selection of this model, in contrast to others such as contusions and complete transections have been discussed elsewhere. ${ }^{[36]}$ All surgical procedures 
were performed under intraperitoneal (IP) analgesia with xylacine $\left(10 \mathrm{mg} \mathrm{kg}^{-1}\right)$ and anesthesia with sodium pentobarbital $\left(55 \mathrm{mg} \mathrm{kg}^{-1}\right)$ mixed with atropine $\left(0.05 \mathrm{mg} \mathrm{kg}^{-1}\right)$. Eyes were covered with Lubrithal ${ }^{\mathrm{TM}}$ gel to prevent corneal abrasion and dehydration. Temperature, color and respiratory frequency were constantly monitored during the complete surgical procedure. A midline incision was performed on the dorsal skin from the base of the skull up to the T3 backbone level. Superficial and deep muscles were carefully dissected, always along the midline. Vertebrae insertions of deeper muscles were cut at both sides of the spinal processes to get access to the backbone (typically from C4 to T2). After thorough identification, C5 vertebra was removed and the meninges visualized. A square incision was practiced on the dura mater to completely expose the spinal cord at C6 level. By using appropriate micro-scissors, three firm cuts were then carried out at C6 (one rostral, one caudal and one sagittal at the midline) to achieve a right lateral hemisection of approximately $8 \mathrm{~mm}^{3}$. Immediately after, fragments of neural tissue cut were removed from the lesion site as much as possible. Bleeding was quickly contained by using small sterile pieces of Spongostan ${ }^{\circledR}$. In animals receiving rGO scaffolds, monoliths of appropriate dimensions were implanted at the lesion site to fill in the cavity created. Superficial and deep muscles were smoothly approximated with a $3 / 0$ synthetic absorbable sterile surgical suture (Coated VICRYL®), also used to close the skin. A plastic bandage (Nobecutan $\left.{ }^{\circledR}\right)$ was finally sprayed on top of the sutured area to facilitate healing and avoid infection. Treatment groups included: control without injury $(\mathrm{n}=6)$, injury $(I, \mathrm{n}=9)$ and injury with implantation of $\mathrm{rGO}$ scaffold $(r G O, \mathrm{n}=11)$. The immediate post-operatory care protocol included subcutaneous administration of antibiotics (enrofloxacine $2.5 \%, 0.3 \mathrm{~mL} \mathrm{kg-1}$ every $24 \mathrm{~h}$ ), analgesia (meloxicam $5 \mathrm{mg}$ $\mathrm{mL}^{-1}, 0.1 \mathrm{~mL} \mathrm{~kg}^{-1}$, every $\left.12 \mathrm{~h}\right)$ and saline solution $(\mathrm{NaCl} 0.9 \%, 5 \mathrm{~mL}$, once or twice a day depending on the hydration state of the animal) during the first three days. Posteriorly, 
animals were frequently observed with major attention placed into signs of pain, distress, dehydration, intestinal obstruction, and respiratory failure. After 4 months of implantation (120 DPI), animals were sacrificed by using a standard perfusion-fixation protocol and the thoraco-abdominal viscerae and spinal cords were extracted. All organs were examined macroscopically looking for any signs of gross damage before histological processing. In rats dedicated to TEM studies (control, $\mathrm{n}=1 ; I, \mathrm{n}=3 ; r G O, \mathrm{n}=3$ ), a specific perfusion-fixation procedure with a circulating solution containing paraformaldehyde at $4 \%$ and glutaraldehyde at $1 \%$ in phosphate buffer was conducted. Behavioral tests: The degree of forelimb motor behavior compromise and its evolution over time was assessed by two different behavioral tests: grooming and forelimb asymmetry. Forelimb grooming function was assessed by using a scoring system. The forelimb asymmetry test allows detecting asymmetries in the paw use preference of the animal exploratory behavior caused by CNS injuries. For further experimental details and references on the behavioral tests used, please refer to the Supplementary Material. Atomic force microscopy and force spectroscopy: AFM measurements were performed by using a commercial instrument (JPK Nanowizard 3, JPK Instruments AG, Berlin, Germany) mounted on a Axio Observer A1 inverted microscope (Carl Zeiss, Oberkochen, Germany). Depending on the biological sample (cells or tissues), different types of cantilevers were used. Force-distance curves were acquired to determine the Young's modulus of neural cells and spinal cord tissues. Embryonic neural progenitor cells for AFM testing of individual neural cells were gently provided by the Hospital Nacional de Parapléjicos (Toledo, Spain). For further experimental details on AFM studies, please refer to the Supplementary Material.

Magnetic resonance imaging (MRI) studies: The fragment between $\mathrm{C} 4$ and $\mathrm{T} 1$, which contains the main spinal cord structures involved in the rat anterior limb function, was 
prepared for MRI analysis. MRI images were acquired in a 7T Bruker Biospin MRI machine (Ettlingen, Germany) BioSpec 70/30. The different measurements were made using images of a turbo spin echo sequence enhanced in T2 (RARE turbo) and axial acquisition. For further experimental details on MRI studies, please refer to Supplementary Material.

Histological studies: All perfused tissue samples were placed in paraformaldehyde $4 \%$ at $4{ }^{\circ} \mathrm{C}$ overnight and then 3 days in sucrose (30\% in phosphate buffer saline, PBS) at $4{ }^{\circ} \mathrm{C}$ for cryo-protection. Tissue pieces were mounted on plastic containers, quick-frozen in Optimal Cutting Temperature compound (Tissue Tek, Hatfield, PA) and cut in horizontal sections of $10-\mu \mathrm{m}$ by using a Leica CM1900 cryostat with an angle of $10^{\circ}$. In the particular case of spinal cords, the entire C5-C7 fragment was cut in sagittal sections from right to left. Organs were stained with conventional hematoxylin-eosin (HE) stain. Spinal cords were examined after Masson's trichrome for visualizing collagen. In all cases, panoramic images at low magnification were collected by using an Olympus BX61 microscope.

TEM studies of rGO implants and organs: Samples dedicated to TEM studies were additionally post-fixed in osmic tetroxide (1\% in distilled water) for $1 \mathrm{~h}$. Dehydration was then followed by immersion in successive solutions of ethanol at increasing concentrations $(30,50,70,95$, and $100 \%)$, with a final step in pure acetone. Samples were then included in Durcupán resin by consecutive steps at increasing concentrations $\left(1: 3,1: 1,3: 1\right.$ in acetone). Final samples in pure resin were polymerized at $60{ }^{\circ} \mathrm{C}$ for $48 \mathrm{~h}$. Ultrathin sections $(c a .60 \mathrm{~nm})$ were obtained and subsequently stained with uracil acetate and lead citrate. The visualization was carried out by using a Jeol JEM 1010 microscope at $80-100 \mathrm{kV}$. Images were processed by using the Fiji software for quantitative estimations. Features of rGO dissociation were estimated by analyzing the distribution of 
pixels in the grey scale from images captured at $300,000 \mathrm{X}$ at $100 \mathrm{kV}$ in similar conditions of brightness ( $\mathrm{N} \geq 25$ from 3 different animals per group). Cavities at the lesion site and tissue infiltration degree were estimated by studying the distribution of pixels in the grey scale from images captured at $2,000 \mathrm{X}$ at $80 \mathrm{kV}$ in similar conditions $(\mathrm{N} \geq 20$ from 3 different animals per group).

Immunofluorescence studies: Specific details for each of the antibodies used in this study are summarized in Table S2. Spinal cord samples were examined for the presence of the following markers: (1) map-2 for somas and dendrites in neurons, (2) tau for axons in neurons, (3) vimentin for non-neuron cells including glial and connective tissue cells, (4) glial fibrillary acidic protein (GFAP) for astrocytes, (5) ED1 for macrophages, (6) platelet-derived growth factor receptor $\beta$ (PDGFR $\beta$ ) for pericytes, precursors of oligodendrocytes and connective tissue cells such as fibroblasts, (7) SMI-311 for cytoskeletal neurofilaments in neurites, (8) $\beta$-III tubulin for cytoskeleton microtubules in neurites, (9) RECA-1 for endothelial cells in blood vessels, (10) laminin for basement membranes in blood vessels, (11) vesicular glutamate transporter 2 (Vglut2) for excitatory glutamatergic fibers, (12) tyrosine hydroxylase for excitatory dopaminergic fibers, and (13) serotonin for inhibitory serotoninergic fibers. Appropriate secondary antibodies were selected accordingly. In all cases, cell nuclei were visualized by labeling with Hoechst $\left(1 \mathrm{mg} \mathrm{mL}^{-1}\right)$. Fluorescence images were collected by using a Leica TCS SP5 microscope. Capture conditions were fixed by using sections from the three experimental groups incubated with the secondary antibodies but without the primary ones. All images were thereafter captured under these conditions. All fluorescence images were automatically quantified by using a customized macro in Fiji software as the number of pixels (correspondent $\mu \mathrm{m}^{2}$ ) positively stained for each particular fluorescence marker after the definition of the correspondent threshold of positive labeling. Control spinal 
cords and contralateral hemicords in injured animals served as reference values to define correspondent threshold values for each marker. At least three non-overlapping images at 40X per animal were acquired in each position of interest $(\mathrm{N} \geq 9$ per fluorescence marker, study region and group from at least 3 different animals per group). Areas under study were: left hemicord - LH-, perilesional areas at 1-2 mm from the lesion site - PL12-, caudal interface of the lesion - CIF-, rostral interface of the lesion - RIF-, lesion site without scaffold, and lesion site with scaffold. Additional bright field images were taken to define scaffold location.

Statistics: Values were expressed as the mean \pm standard deviation of at least three different animals per group $(\mathrm{N} \geq 3)$. In the box plots, the line and square inside correspond to the median and the mean of the distribution, respectively. The top and bottom of the box represent, respectively, the 75 th and 25 th percentiles. The whiskers out of the box denote the range of outer-most data that fall within $1.5 \mathrm{x}$ interquartile range. Additionally, single data points were overlapped with their correspondent box plots. Statistical analysis was performed by using the Statistical Package for the Social Sciences (SPSS, version 17.0), except for AFM measurements that were analyzed by using the Origin platform (OriginLab). Comparisons among groups were done by one-way analysis of variance (ANOVA) and either post-hoc Scheffé or Games-Howell tests (homogeneous vs. heterogeneous variances, respectively, as dictated by Levene's test). For comparisons between two independent groups, a T-test was used. MRI data were compared by using the Mann Whitney Rank sum test. In all cases, the significance level was defined as $\mathrm{p}<$ 0.05 .

\section{Acknowledgements}

This work was supported by the Ministerio de Economía y Competitividad and the Fondo Europeo de Desarrollo Regional (MAT2016-78857-R, MAT2016-76507-R and 
MAT2016-80394-R, MINECO/FEDER, UE) and the European Research Council ERCAdG-340177 (3DNanoMech). Authors are grateful to Dr. André Espinha for his assistance with figure preparation and formatting. Dr. Daniel García-Ovejero from the Hospital Nacional de Parapléjicos (HNP) is acknowledged for help with sectioning of fresh spinal cords by vibratome. Dr. Jose Ángel Rodríguez and Dr. Javier Mazarío from the Service of Microscopy and Image Analysis at the HNP are acknowledged for assistance with CLSM studies and Dr. Fernando García-García and Dr. Marina Benito from the HNP for assistance with MRI studies. Authors are thankful to Dr. Enrique Rodríguez, Dr. Francisco Urbano and Dr. Covadonga Agudo from the Servicio Interdepartamental de Investigación at the Universidad Autónoma de Madrid for respective assistance with SEM and TEM.

\section{Data availability}

The raw and processed data required to reproduce these findings will be available to download from DIGITAL.CSIC upon acceptance. 


\section{References}

[1] S. Y. Park, J. Park, S. H. Sim, M. G. Sung, K. S. Kim, B. H. Hong, S. Hong, Enhanced differentiation of human neural stem cells into neurons on graphene. Adv. Mater. 2011, 23, $\mathrm{H} 263$.

[2] S. Marchesan, L. Ballerini, M. Prato, Nanomaterials for stimulating nerve growth. Science 2017, 356, 1010.

[3] Y. Wang, W. C. Lee, K. K. Manga, P. K. Ang, J. Lu, Y. P. Liu, C. T. Lim, K. P. Loh, Fluorinated graphene for promoting neuro-induction of stem cells. Adv. Mater. 2012, 24, 4285.

[4] N. Li, X. Zhang, Q. Song, R. Su, Q. Zhang, T. Kong, L. Liu, G. Jin, M. Tang, G. Cheng, The promotion of neurite sprouting and outgrowth of mouse hippocampal cells in culture by graphene substrates. Biomaterials 2011, 32, 9374.

[5] M. Tang, Q. Song, N. Li, Z. Jiang, R. Huang, G. Cheng, Enhancement of electrical signaling in neural networks on graphene films. Biomaterials 2013, 34, 6402.

[6] R. Rauti, N. Lozano, V. León, D. Scaini, M. Musto, I. Rago, F. P. Ulloa Severino, A. Fabbro, L. Casalis, E. Vázquez, K. Kostarelos, M. Prato, L. Ballerini, Graphene oxide nanosheets reshape synaptic function in cultured brain networks. ACS Nano 2016, 10, 4459.

[7] N. P. Pampaloni, M. Lottner, M. Giugliano, A. Matruglio, F. D‘Amico, M. Prato, J. A. Garrido, L. Ballerini, D. Scaini, Single-layer graphene modulates neuronal communication and augments membrane ion currents. Nat. Nanotechnol. 2018. DOI:. 10.1038/s41565-018-0163-6.

[8] Y. Qian, X. Zhao, Q. Han, W. Chen, H. Li, W. Yuan, An integrated multi-layer 3Dfabrication of PDA/RGD coated graphene loaded PCL nanoscaffold for peripheral nerve restoration. Nat. Commun. 2018, 9, 323. 
[9] C. Defterali, R. Verdejo, L. Peponi, E. D. Martín, R. Martínez-Murillo, M. Á. LópezManchado, C. Vicario-Abejón, Thermally reduced graphene is a permissive material for neurons and astrocytes and de novo neurogenesis in the adult olfactory bulb in vivo. Biomaterials 2015, 82, 84 .

[10] K. E. Kitko, T. Hong, R. M. Lazarenko, D. Ying, Y. Q. Xu, Q. Zhang, Membrane cholesterol mediates the cellular effects of monolayer graphene substrates. Nat. Commun. 2018, 9, 796.

[11] A. Bianco, Graphene: Safe or toxic? The two faces of the medal. Angew. Chem. Int. Ed. 2013, 52, 4986.

[12] G. Reina, J. M. González-Domínguez, A. Criado, E. Vázquez, A. Bianco, M. Prato, Promises, facts and challenges for graphene in biomedical applications. Chem. Soc. Rev. 2017, 46, 4400 .

[13] Y. Li, L. Feng, X. Shi, X. Wang, Y. Yang, K. Yang, T. Liu, G. Yang, Z. Liu, Surface coating-dependent cytotoxicity and degradation of graphene derivatives: Towards the design of non-toxic, degradable nano-graphene. Small 2014, 10, 1544.

[14] R. Kurapati, S. P. Mukherjee, C. Martín, G. Bepete, E. Vázquez, A. Pénicaud, B. Fadeel, A. Bianco, Degradation of single-layer and few-layer graphene by neutrophil myeloperoxidase. Angew. Chem. Int. Ed. Engl. 2018. DOI:.10.1002/anie.201806906.

[15] R. Kurapati, J. Russier, M. A. Squillaci, E. Treossi, C. Ménard-Moyon, A. E. Del Río-Castillo, E. Vazquez, P. Samorí, V. Palermo, A. Bianco, Dispersibility-dependent biodegradation of graphene oxide by myeloperoxidase. Small 2015, 11, 3985.

[16] D. Guarnieri, P. Sánchez-Moreno, A. E. Del Rio Castillo, F. Bonaccorso, F. Gatto, G. Bardi, C. Martín, E. Vázquex, T. Catelani, S. Sabella, P. P. Pompa, Biotransformation and biological interaction of graphene and graphene oxide during simulated oral ingestion. Small 2018. DOI: 10.1002/smll.201800227. 
[17] C. Göritz, D. O. Dias, N. Tomilin, M. Barbacid, O. Shupliakov, J. Frisén, A pericyte origin of spinal cord scar tissue. Science 2011, 333, 238.

[18] E. Moeendarbary, I. P. Weber, G. K. Sheridan, D. E. Koser, S. Soleman, B. Haenzi, E. J. Bradbury, J. Fawcett, K. Franze, The soft mechanical signature of glial scars in the central nervous system. Nat. Commun. 2017, 8, 14787.

[19] M. M. Pathak, J. L. Nourse, T. Tran, J. Hwe, J. Arulmoli, D. T. Le, E. Bernardis, L. A. Flanagan, F. Tombola, Stretch-activated ion channel Piezo1 directs lineage choice in human neural stem cells. Proc. Natl. Acad. Sci. U.S.A. 2014, 111, 16148.

[20] D. Koch, W. J. Rosoff, J. Jiang, H. M. Geller, J. S. Urbach, Strength in the periphery: Growth cone biomechanics and substrate rigidity response in peripheral and central nervous system neurons. Biophys. J. 2012, 102, 452.

[21] P. Moshayedi, F. Costa Lda, A. Christ, S. P. Lacour, J. Fawcett, J. Guck, K. Franze, Mechanosensitivity of astrocytes on optimized polyacrylamide gels analyzed by quantitative morphometry. J. Phys. Condens. Matter. 2010, 22, 194114.

[22] National Spinal Cord Injury Statistical Center, Facts and Figures at a Glance. Birmingham, AL: University of Alabama at Birmingham, 2015. https://www.nscisc.uab.edu/Public/Facts\%20and\%20Figures\%20-\%202018.pdf. Last retrieved: 07/31/2018.

[23] A. de los Reyes-Guzmán, E. López-Dolado, V. Lozano-Berrio, S. Pérez-Nombela, D. Torricelli, J. L. Pons, A. Gil-Agudo, Upper limb electromyographic analysis synchronized with kinematics in cervical spinal cord injured patients during the activity of daily living of drinking. JSM Physical. Med. Rehabil. 2017, 1, 1004.

[24] J. E. Collazos-Castro, V. M. Soto, M. Gutiérrez-Davila, M. Nieto-Sampedro, Motoneuron loss associated with chronic locomotion impairments after spinal cord contusion in the rat. J. Neurotrauma 2005, 22, 544. 
[25] J. F. Ditunno, Spinal shock revisited: a four-phase model. Spinal Cord 2004, 42, 383.

[26] I. R. Minev, P. Musienko, A. Hirsch, Q. Barraud, N. Wenger, E. M. Moraud, J. Gandar, M. Capogrosso, T. Milekovic, L. Asboth, R. F. Torres, N. Vachicouras, Q. Liu, N. Pavlova, S. Duis, A. Larmagnac, J. Vörös, S. Micera, Z. Suo, G. Courtine, S.P. Lacour, Electronic dura mater for long-term multimodal neural interfaces. Science 2015, 347, 159. [27] R. Van der Brand, J. Heutschi, Q. Barraud, J. DiGiovanna, K. Bartholdi, M. Huerlimann, L. Friedli, I. Vollenweider, E. M. Moraud, S. Duis, N. Dominici, S. Micera, P. Musienko, G. Courtine, Restoring voluntary control of locomotion after paralyzing spinal cord injury. Science 2012, 336, 1182.

[28] T. Führmann, M. S. Shoichet, The role of biomaterials in overcoming barriers to regeneration in the central nervous system. Biomed. Mater. 2018, 13, 050201.

[29] T. Führmann, P. N. Anandakumaran, M. S. Shoichet, Combinatorial therapies after spinal cord injury: How can biomaterials help? Adv. Healthc. Mater. 2017, 6. DOI: 10.1002/adhm.201601130.

[30] A. E. Haggerty, I. Maldonado-Lasunción, M. Oudega, Biomed. Mater. 2018, 13, 044105 .

[31] J. R. Slotkin, C. D. Pritchard, B. Luque, J. Ye, R. T. Layer, M. S. Lawrence, T. M. O’Shea, R. R. Roy, H. Zhong, I. Vollenweider, V. R. Edgerton, G. Courtine, E. J. Woodard, R. Langer, Biodegradable scaffolds promote tissue remodeling and functional improvement in non-human primates with acute spinal cord injury. Biomaterials 2017, $123,63$.

[32] L. T. A. Hong, Y. M. Kim, H. H. Park, D. H. Hwang, Y. Cui, E. M. Lee, S. Yahn, J. K. Lee, S. C. Song, B. G. Kim, An injectable hydrogel enhances tissue repair after spinal cord injury by promoting extracellular matrix remodeling. Nat. Commun. 2017, 8, 533. 
[33] J. S. Rao, C. Zhao, A. Zhang, H. Duan, P. Hao, R. H. Wei, J. Shang, W. Zhao, Z. Liu, J. Yu, K. S. Fan, Z. Tian, Q. He, W. Song, Z. Yang, Y. E. Sun, X. Li, NT3-chitosan enables de novo regeneration and functional recovery in monkeys after spinal cord injury. Proc. Nat. Acad. Sci. U.S.A. 2018, 115, E5595.

[34] S. Usmani, E. R. Aurand, M. Medelin, A. Fabbro, D. Scaini, J. Laishram, F. B. Rosselli, A. Ansuini, D. Zoccolan, M. Scarselli, M. De Crescenzi. S. Bosi, M. Prato, L. Ballerini, 3D meshes of carbon nanotubes guide functional reconnection of segregated spinal explants. Sci. Adv. 2016, 2, e1600087.

[35] A. Fabbro, A. Villari, J. Laishram, D. Scaini, F. M. Toma, A. Turco, M. Prato, L. Ballerini, Spinal cord explants use carbon nanotube interfaces to enhance neurite outgrowth and to fortify synaptic inputs. ACS Nano 2012, 6, 2041.

[36] E. López-Dolado, A. González-Mayorga, M. T. Portolés, M. J. Feito, M. L. Ferrer, F. Del Monte, M. C. Gutiérrez, M. C. Serrano, Subacute tissue response to 3D graphene oxide scaffolds implanted in the injured rat spinal cord. Adv. Healthc. Mater. 2015, 4, 1861.

[37] E. López-Dolado, A. González-Mayorga, M. C. Gutiérrez, M. C. Serrano, Immunomodulatory and angiogenic responses induced by graphene oxide scaffolds in chronic spinal hemisected rats. Biomaterials 2016, 99, 72.

[38] Y. B. Lu, K. France, G. Seifert, C. Steinhäuser, F. Kirchhoff, H. Wolburg, J. Guck, P. Janmey, E-Q. Wei, J. Käs, A. Reichenbach, Viscoelasctic properties of individual glial cells and neurons in the CNS. Proc. Natl. Acad. Sci. U.S.A. 2006, 103, 17759.

[39] F. Della Pellea, R. Di Battistaa, L. Vázquez, F. J. Palomares, M. Del Carlo, M. Sergi, D. Compagnone, A. Escarpa, Press-transferred carbon black nanoparticles for classselective antioxidantelectrochemical detection. Appl. Mater. Today 2017, 9, 29. 
[40] P. D. Garcia, R. Garcia, Determination of the elastic moduli of a single cell cultured on a rigid support by force microscopy. Biophys. J. 2018, 114, 2923.

[41] P. D. Garcia, C. R. Guerrero, R. Garcia, Time-resolved nanomechanics of a single cell under the depolymerization of the cytoskeleton. Nanoscale 2017, 9, 12051.

[42] J. Kiernan, Formaldehyde, formalin, paraformaldehyde and glutaraldehyde: what they are and what they do. Micros. Today 2000, 12, 8.

[43] S. Kubinová, D. Horák, A. Hejcl, Z. Plichta, J. Kotek, V. Proks, S. Forostyak, E. Syková. SIKVAV-modified highly superporous PHEMA scaffolds with oriented pores for spinal cord injury repair. J. Tissue Eng. Regen. Med. 2015, 9, 1298.

[44] S. Kubinová, D. Horák, A. Hejčl, Z. Plichta, J. Kotek, E. Syková, Highly superporous cholesterol-modified poly(2-hydroxyethyl methacrylate) scaffolds for spinal cord injury repair. J. Biomed. Mater. Res. Part A 2011, 99, 618.

[45] E. C. Tsai, P. D. Dalton, M. S. Shoichet, C. H. Tator, Synthetic hydrogel guidance channels facilitate regeneration of adult rat brainstem motor axons after complete spinal cord transection. Biomaterials 2006, 27, 519.

[46] D. Shahriari, J. Y. Koffler, M. H. Tuszynski, W. M. Campana, J. S. Sakamoto, Hierarchically ordered porous and high-volume polycaprolactone microchannel scaffolds enhanced axon growth in transected spinal cords. Tissue Eng. Part A 2017, 23, 415.

[47] N. A. Silva, A. J. Salgado, R. A. Sousa, J. T. Oliveira, A. J. Pedro, H. Leite-Almeida, R. Cerqueira, A. Almeida, F. Mastronardi, J. F. Mano, N. M. Neves, N. Sousa, R. L. Reis, Development and characterization of a novel hybrid tissue engineering-based scaffold for spinal cord injury repair. Tissue Eng. Part A 2010, 16, 45.

[48] J. E. McKenna, G. T. Prusky, I. Q. Whishaw, Cervical motoneuron topography reflects the proximocaudal organization of muscles and movements of the rat forelimb. A retrograde carbocyanine dye analysis. J. Comp. Neurol. 2000, 419, 286. 
[49] E. López-Dolado, A. Lucas-Osma, J. E. Collazos-Castro, Dynamic motor compensations with permanent, focal loss of forelimb force after cervical spinal cord injury. J. Neurotrauma 2013, 30, 191.

[50] M. Itzkovich, H. Shefler, L. Front, R. Gur-Pollack, K. Elkayam, V. Bluvshtein, I. Gelernter, A. Catz, (Spinal Cord Independence Measure version III): Reliability of assessment by interview and comparison with assessment by observation. Spinal Cord 2018, 56, 46.

[51] L. Filli, B. Zöner, O. Weinmann, M. E. Schwab, Motor deficits and recovery in rats with unilateral spinal cord hemisection mimic the Brown-Sequard syndrome. Brain 2011, $134,2261$.

[52] A. de los Reyes-Guzmán, A. Gil-Agudo, B. Peñasco-Martín, M Solís-Mozos, A. del Ama-Espinosa, E. Pérez-Rico, Kinematic analysis of the daily activity of drinking from a glass in a population with cervical spinal cord injury. J. Neuroeng. Rehabil. 2010, 7, 41.

[53] J. W. Austin, M. Afshar, M. G. Fehlings, The relationship between localized subarachnoid inflammation and parenchymal pathophysiology after spinal cord injury. $J$ Neurotrauma 2012, 29, 1838.

[54] Y. Karam, P. W. Hitchon, N. E. Mhanna, W. He, J. Noeller, Post-traumatic syringomyelia: Outcome predictors. Clin. Neurol. Neurosurg. 2014, 124, 44.

[55] L. Zhou, L. Fan, X. Yi, Z. Zhou, C. Liu, R. Fu, C. Dai, Z. Wang, X. Chen, P. Yu, D. Chen, G. Tan, Q. Wang, C. Ning, Soft conducting polymer hydrogels cross-linked and doped by tannic acid for spinal cod injury repair. ACS Nano 2018, DOI 10.1021/acsnano.8b04609.

[56] J. T. Yabe, W. K. Chan, F. S. Wang, A. Pimenta, D. D. Ortiz, T. B. Shea, Regulation of the transition from vimentin to neurofilaments during nneuronal differentiation. Cell Motil. Cytoskeleton 2003, 56, 193. 
[57] S. A. Moody, M. S. Quigg, A. Frankfurter, The development of the peripheral trigeminal system in the chick embryo revealed by an isotype specific anti- $\beta$-tubulin monoclonal antibody. J. Comp. Neurol. 1989, 279, 567.

[58] M. K. Lee, J. B. Tuttle, L. I. Rebhun, D. W. Cleveland, A. Frankfurter, The expression and posttranslational modification of a neuronspecific $\beta$-tubulin isotype during chick embryogenesis. Cell Motil. Cytoskeleton 1990, 17, 118.

[59] D. Panda, H. P. Miller, A. Banerjee, R. F. Luduena, L. Wilson, Microtubule dynamics in vitro are regulated by the tubulin isotype composition. Proc. Natl. Acad. Sci. U.S.A. 1994, 91, 11358.

[60] L. Filli, A. K. Engmann, B. Zörner, O. Weinmann, T. Moraitis, M. Gullo, H. Kasper, R. Schneider, M. E. Schwab, Bridging the gap: A reticulo-propiospinal detour bypassing an incomplete spinal cord injury. J. Neurosci. 2014, 34, 13399.

[61] M. Ballerman, K. Fouad, Spontaneous locomotor recovery in spinal cord injured rats accompanied by anatomical plasticity of reticulospinal fibers. Eur. J. Neurosci. 2006, 23, 1988.

[62] J. R. Flynn, B. A. Graham, M. P. Galea, R. J. Callister, The role of propiospinal interneurons in recovery from spinal cord injury. Neuropharmacology 2011, 60, 809.

[63] G. Courtine, B. Song, R. R. Roy, H. Zhong, J. E. Herrmann, Y. Ao, J. Qi, V. R. Edgerton, M. V. Sofroniew, Recovery of supraspinal control of stepping via indirect propiospinal relay connections after spinal cord injury. Nat. Med. 2008, 14, 69.

[64] N. Nishimura, Y. Morichika, T. Isa, A subcortical oscillatory network contributes to recovery of hand dexterity after spinal cord injury. Brain 2009, 132, 709.

[65] M. S. Esposito, P. Capelli, S. Arber, Brainstem nucleus MdV mediates skilled forelimb motor tasks. Nature 2014, 508, 351. 
[66] M. Hägglund, L. Borgius, K. J. Dougherty, O. Kiehn, Activation of groups of excitatory neurons in the mammalian spinal cord or hindbrain evokes locomotion. Nat. Neurosci. 2010, 13, 246.

[67] B. Chen, J. He, H. Yang, Q. Zhang, L. Zhang, X. Zhang, E. Xie, C. Liu, R. Zhang, Y. Wang, L. Huang, D. Hao, Repair of spinal cord injury by implantatoin of bFGFincorporated HEMA-MOETACL hydrogel in rats. Sci. Rep. 2015, 5, 9017.

[68] Y. Wen, S. Yu, Y. Wu, R. Ju, H. Wang, Y. Liu, y. Wang, Q. Xu, Spinal cord injury repair by implantatin of structured hyaluronic acid scaffold with PLGA microspheres in the rat. Cell Tissue Res. 2015, DOI 10.1007/s00441-015-2298-1.

[69] E. R. Burnside, E. J. Bradbury, Manipulating the extracellular matrix and its role in brain and spinal cord plasticity and repair. Neuropathol. Appl. Neurobiol. 2014, 40, 26.

[70] L. R. Nih, S. Gojgini, S. T. Carmichael, T. Segura, Dual-function injectable angiogenic biomaterial for the repair of brain tissue following stroke. Nat. Mater. 2018. DOI: $10.1038 / \mathrm{s} 41563-018-0083-8$.

[71] A. Campana, T. Cramer, D. T. Simon, M. Berggren, F. Biscarini, Electrocardiographic recording with conformable organic electrochemical transistor fabricated on resorbable bioscaffold. Adv. Mater. 2014, 26, 3874.

[72] S. P. Mukherjee, A. R. Gliga, B. Lazzaretto, B. Brandner, M. Fielden, C. Vogt, L. Newman, A. F. Rodrigues, W. Shao, P. M. Fournier, M. S. Toprak, A. Star, K. Kostarelos, K. Bhattacharya, B. Fadeel. Nanoscale 2018, 10, 1180.

[73] H. Yue, W. Wei, Z. Yue, B. Wang, N. Luo, Y. Gao, D. Ma, G. Ma, Z. Su, The role of the lateral dimension of graphene oxide in the regulation of cellular responses. Biomaterials 2012, 33, 4013. 
[74] Q. Mu, G. Su, L. Li, B. O. Gilbertson, L. H. Yu, Q. Zhang, Y. P. Sun, B. Yan, Sizedependent cell uptake of protein-coated graphene oxide nanosheets. ACS Appl. Mater. Interfaces 2012, 4, 2259.

[75] K. Wang, J. Ruan, H. Song, J. Zhang, Y. Wo, S. Guo, D. Cui, Biocompatibility of graphene oxide. Nanoscale Res. Lett. 2011, 6, 1.

[76] X. Zhang, J.Yin, C. Peng, W. Hu, Z. Zhu, W. Li, C. Fan, Q. Huang, Distribution and biocompatibility studies of graphene oxide in mice after intraveneous administration. Carbon 2011, 49, 986.

[77] J. H. Liu, S. T. Yang, H. Wang, Y. Chang, A. Cao, Y. Liu, Effect of size and dose on the biodistribution of graphene oxide in mice. Nanomedicine (Lond) 2012, 7, 1801. 